\title{
Suppression of beam-ion instability in electron rings with multibunch train beam fillings
}

\author{
L. Wang, Y. Cai, and T. O. Raubenheimer \\ SLAC, 2575 Sand Hill Road, Menlo Park, California 94025, USA \\ H. Fukuma \\ KEK, 1-1 Oho, Tsukuba, Ibaraki 305-0801, Japan \\ (Received 30 November 2010; published 17 August 2011)
}

\begin{abstract}
The ion-caused beam instability in the future light sources and electron damping rings can be serious due to the high beam current and ultrasmall emittance of picometer level. One simple and effective mitigation of the instability is a multibunch train beam filling pattern which can significantly reduce the ion density near the beam, and therefore reduce the instability growth rate up to 2 orders of magnitude. The suppression is more effective for high intensity beams with low emittance. The distribution and the electric field of trapped ions are benchmarked to validate the model used in the paper. The wakefield of ion cloud and the beam-ion instability is investigated both analytically and numerically. We derived a simple formula for the buildup of ion cloud and instability growth rate with the multibunch train filling pattern. The simulation in NSLSII, PEPX, SuperKEKB, and the observation in SPEAR3 are used to compare with our analyses. The analyses agree well with simulations and observations.
\end{abstract}

DOI: $10.1103 /$ PhysRevSTAB.14.084401

PACS numbers: 29.27.Bd, 29.20.db

\section{INTRODUCTION}

In accelerators with negatively charged beams, an electron accelerator for instance, ions generated from the residual gas molecules can be trapped by the beam. The trapped ions interact resonantly with the beam and cause a beam-ion coherent instability, tune shift, and emittance blowup [1-3]. This beam-ion instability can occur in both ring and linac [4]. Besides the electron accelerator, the beam-ion instability can also occur in other negatively charged beams, including the antiproton beam [5]. This paper studies the beam-ion instability in an electron ring only. A similar method can be used to study the instability in a linac.

"Conventional" ion effects occur when ions are trapped by a circulating electron beam for multiple revolutions. To avoid conventional ion trapping, a long gap is introduced in the electron beam. However, such a gap does not preclude ions from accumulating during one passage of the single bunch-train beam, and those ions can still cause a fast ion instability (FII) [6-9], especially in long storage rings where a single bunch train can be long enough to trap enough ions and cause FII.

Beam shaking is an effective way to make the ion unstable [10]. However, the required shaking amplitude should be larger than the beam size. Such a disturbed beam cannot be used. The alternate way to avoid trapped ions is to introduce clearing electrodes. A minimum condition for

Published by the American Physical Society under the terms of the Creative Commons Attribution 3.0 License. Further distribution of this work must maintain attribution to the author(s) and the published article's title, journal citation, and DOI. the capture of the passing ion is that the transverse field potential provided by the electrode equals the maximum beam's space charge potential, which is typically more than $1 \mathrm{kV}$. Because a clearing electrode can only clear ions nearby, the required number of clearing electrodes is large for a long storage ring. Furthermore, the clearing electrode complicates the mechanical design of the vacuum chamber and also contributes to the machine impedance [11]. For a small ring, this is an effective mitigation; for instance, the beam position monitor can be used to clear ions.

This paper studies the suppression of beam-ion instability by simply using multibunch train filling patterns. The instability growth rate is proportional to the ion density at the beam as shown later. Therefore, it is important to reduce the ion density in the vicinity of the beam to a tolerable level. A single bunch-train filling pattern is used in B-factory and most existing light sources. Numerical studies show that a multibunch train with short gaps is very effective at reducing the ion density near the beam [12]. The extension of the simulation study can be found in $[13,14]$. These short gaps in the filling pattern lead to an absence of focusing forces for the ions every time the gaps travel around the ring. The beam force overfocuses ions and permits them to oscillate to large amplitudes out of the beam center. However, no analysis has been done to understand the mechanism. It is practically important to have the analysis done to guide the operation on how to choose the beam filling pattern.

This study focuses on the physical understanding, instead of simulation. The example of the numerical and experimental results for NSLSII, PEPX, SuperKEKB, and SPEAR3 are used to compare with our analysis. 
Although our approaches are similar to the one used in the study of electron cloud and instability driven by traditional impedance, it is far from being a mere repetition of already known results. In fact, our analysis will rove superior when it comes to physical understanding and accuracy of approximation: it has important application in estimation of practical quantities.

This paper systematically studies beam instability with multibunch train beam filling. The simple physical model considered in this paper gives simple analytical relations. Such a model allows one to obtain a deeper insight into the physics of beam-ion instability with a general beam filling pattern. This paper is organized as follows. The transverse distribution and the electric field of trapped ion are investigated in Sec. II. In Sec. III, we describe the interaction force between the electron beam and ions using conventional wake and give the instability growth rate for uniform and single bunch-train filled beam, respectively. The mechanism of the mitigation of beam instability by using multibunch train beam filling pattern is investigated in great detail in Sec. IV. Simple formulas for the buildup of ion cloud and instability growth rate are given. Simulations of the beam-ion instability in NSLSII, PEPX, and SuperKEKB and the observations in SPEAR3 are presented to compare with the analyses. The ion cloud induced tune shift is briefly discussed in Sec. V. Finally, in Sec. IV we come to conclusions.

\section{TRANSVERSE DISTRIBUTION AND FIELD OF AN ION CLOUD}

It is important to investigate the distribution and the field of trapped ions first. A few works about the ion distribution have been done [15] comparing a larger number of publications in the instabilities [6-9]. For completeness, we briefly analyze the distribution of trapped ions in steady status in the Appendix. One can refer to the early work of Tavares for more detail [15].

The one-dimensional transverse distribution of ions trapped by the electron bunches at the equilibrium state is

$$
\rho(x)=\frac{1}{\pi \sqrt{2 \pi} \sigma_{e, x}} e^{-\left(x^{2} / 4 \sigma_{e, x}^{2}\right)} K_{0}\left(\frac{x^{2}}{4 \sigma_{e, x}^{2}}\right),
$$

where $K_{0}$ is the modified Bessel function of the second kind, and $\sigma_{e}$ is the transverse root mean square (rms) beam size of the electron bunch from which the ions are born. The asymptotic form of ion distribution near the beam center is

$$
\rho(x) \approx-\frac{1}{\pi \sqrt{2 \pi} \sigma_{e, x}} e^{-\left(x^{2} / 4 \sigma_{e, x}^{2}\right)}\left[\ln \left(\frac{x^{2}}{8 \sigma_{e, x}^{2}}\right)+\gamma_{c}\right] .
$$

The same result in Eq. (1) is first given by Tavares [15]. However, the asymptotic form in [15] is incorrect.

Figure 1 compares the ion distributions from analytical and numerical methods. There is an excellent agreement except at the origin where the analytical result diverges.

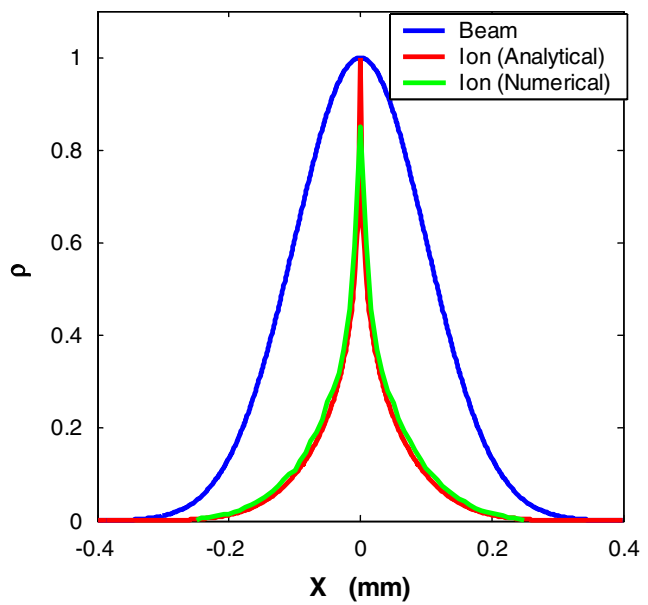

FIG. 1. Comparison of the ion distribution from analytical and numerical approaches. The electron beam profile is also shown in the plot for a better comparison. The rms beam size is $0.1 \mathrm{~mm}$ and $4.5 \mu \mathrm{m}$ in horizontal and vertical planes, respectively.

There is a sharp peak near the center because the ions are sharply focused to that point and the size of the ion cloud is smaller than that of the electron bunch. The result given in Eqs. (1) and (2) works in the linear regime. Surprisingly, it agrees well with simulation, probably because most ions are focused near the beam center where the linear model works. Figure 2 shows the two-dimensional distribution of ion cloud by simulation. Note that the transverse position in the figure is in the unit of beam size. The transverse distribution of ion cloud is very flat for most light sources due to a small coupling.

Since the distribution of the ion cloud is special, there is no available formula to calculate the field of such distributed charged particles. The formula for calculating electric field of a Gaussian beam is well known and an ion cloud with Gaussian distribution has been assumed in the previous analysis of the beam-ion instability [6-8]. However, this assumption has never been benchmarked. Figure 3 shows a comparison of the electric field of an ion cloud shown in Fig. 2 with the field of a Gaussian distributed ion cloud with

$$
\times 10^{12}
$$

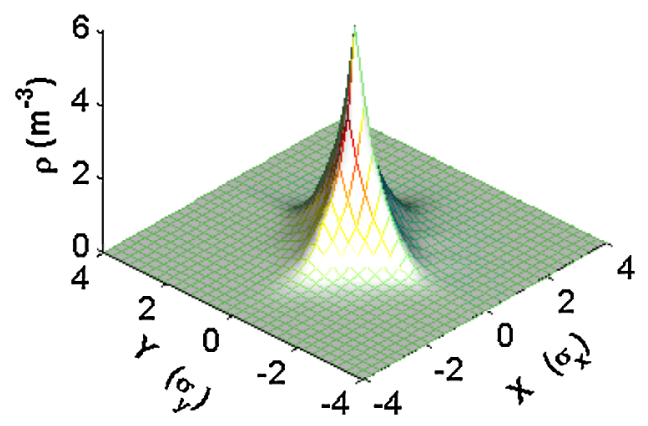

FIG. 2. Two-dimensional distribution of an ion cloud from simulation. $X$ and $Y$ are in the unit of rms beam size 

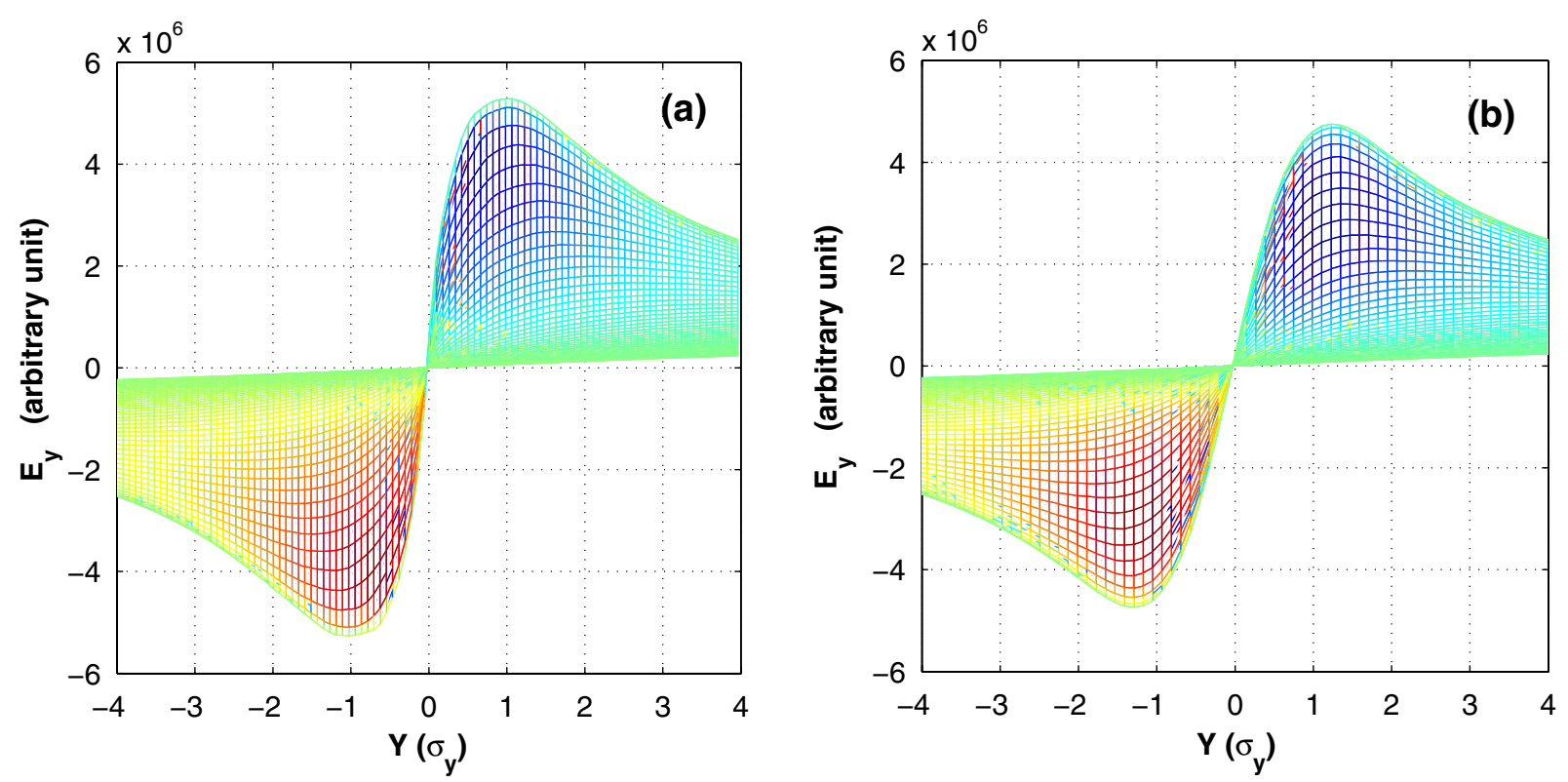

FIG. 3. Vertical electric field of a ion cloud shown in Fig. 2 (a) and a Gaussian distributed ion cloud with $\sigma_{i}=\sigma_{e} / \sqrt{2}$ (b). The total charge is the same for the two distributions. Different lines in the figures are for different $x$. $Y$ is in the unit of rms beam size.

rms size of $\sigma_{i}=\sigma_{e} / \sqrt{2}$. The different lines in Fig. 3 are for different horizontal positions. It is clear that the fields of these two distributions are quite similar overall. The field with a distribution shown in Fig. 2 is slightly stronger and has a larger gradient near the center. In short summary, it slightly underestimates the ion effects by the assumption of a Gaussian distribution with $\sigma_{i}=\sigma_{e} / \sqrt{2}$. This assumption will be used in the rest of this paper. As shown later, the wake and the tune shift due to the trapped ions with this assumption reasonably agree with simulations.

\section{WAKEFIELD AND GROWTH RATE OF THE COUPLED BUNCH INSTABILITY DRIVEN BY IONS}

The two stream instability driven by electron cloud has been studied using the wake model [16]. The distribution of the electron cloud was assumed to be Gaussian in the transverse plane and a coasting beam model was used for the positron beam; however, the ion cloud has special distribution and the electron beam is bunched beam. The wake of ion cloud given in [14] is inaccurate. For instance, the amplitude of the wake is $33 \%$ less than ours and the frequency of the wake also differs from ours and others [6,7]. Reference [14] does not give step-by-step derivation of the wake. It seems that a wrong ion distribution model is used. The distribution of ions is simply assumed to be the same as the electrons. Therefore the wake of ion cloud is well worth careful study and evaluation. In this section we briefly derive the wake due to ions and validate it in great detail with numerical simulation. Then we study the beam-ion instability growth rate using the wake model.
When the charged electron beam passes through the vacuum chamber, ions are generated due to the beam-gas ionization. For a single bunch train consisting of $n_{b}$ bunches followed by a long gap, the ion line density at the tail of the bunch train is given by

$$
\lambda_{i}=\delta_{i} \frac{P}{k T} N_{e} n_{b}
$$

Here $P$ is the vacuum pressure, $\delta_{i}$ is the ionization cross section, $N_{e}$ is the number of electrons per bunch, $T$ is temperature, and $k$ is Boltzmann constant.

We ignore the self-direct space charge effect of the ions and are interested mainly in the coupled motion of the ions and the electron beam. The dipole kick of ion cloud to the electron bunches can be expressed as the direct space charge force $[6,7]$. One alternate approach is to express the kick using a wake function model, which allows the beam-ion instability to be studied in the same way as the coupled bunch instability driven by traditional impedance. When the electron bunches pass through the center of the ion cloud, they are not affected by the ion cloud due to the symmetry of the ion distribution, and the center of mass of the ion cloud does not move. However, if a bunch with a small transverse displacement passes through the cloud, the cloud is disturbed and its center of mass starts to oscillate. Then, the subsequent bunches are deflected by the perturbed ion cloud. The wake force of the ion cloud is calculated as the response to a small displacement of $i$ th bunch $\Delta y_{e 0}$,

$$
W_{y}\left(z_{j}-z_{i}\right)=\frac{\gamma}{N_{e} r_{e}} \frac{\Delta \bar{y}_{e j}^{\prime}}{\Delta y_{e 0}} \quad \text { for } z_{i}<z_{j}
$$


where $r_{e}$ is the classical radius of electron and $\gamma$ is the relativistic factor, and $\Delta y_{e j}^{\prime}$ is the kick of ion cloud to the $j$ th electron bunch.

When the offset $\Delta y_{e 0}$ is smaller than the beam size, the ion cloud receives a linear kick,

$$
\Delta v_{y i}=\frac{N_{e} r_{p} c}{A} \frac{2}{\sum_{y}\left(\sum_{x}+\sum_{y}\right)} \Delta y_{e 0},
$$

where $r_{p}$ is the classical radius of proton, $A$ is the mass number of the ion, $c$ is the speed of light, and the cross sectional area is given by

$$
\sum_{x}=\sqrt{\sigma_{e, x}^{2}+\sigma_{i, x}^{2}}, \quad \sum_{y}=\sqrt{\sigma_{e, y}^{2}+\sigma_{i, y}^{2}}
$$

Here $\sigma_{i}$ is the transverse size of the ion cloud. The kick triggers the oscillation of the ion cloud at amplitude

$$
\hat{y}_{i}=\frac{\Delta v_{y i}}{\omega_{i, y}}=\frac{N_{e} r_{p} c}{\omega_{i, y} A} \frac{2}{\sum_{y}\left(\sum_{x}+\sum_{y}\right)} \Delta y_{e 0} .
$$

Here $\omega_{i, x, y}$ is the oscillation frequency of the ion cloud under the space charge force of the electron beam,

$$
\omega_{i, x, y}=c\left(\frac{2 \lambda_{e} r_{p}}{A k_{x, y} \sigma_{x, y}\left(\sigma_{y}+\sigma_{x}\right)}\right)^{1 / 2} .
$$

Then the following electron bunches receive a kick from the oscillating ion cloud:

$$
\Delta y_{e}^{\prime}=\frac{\Delta v_{y e}}{\gamma c}=\frac{N_{i} r_{e}}{\gamma} \frac{2}{\sum_{y}\left(\sum_{x}+\sum_{y}\right)} y_{i},
$$

where $N_{i}$ is the number of ions. Substituting Eqs. (7) and (9) into Eq. (4), the maximum kick received by the electron bunches gives the amplitude of the wake,

$$
\begin{aligned}
\hat{W}_{y} & =\frac{2}{k_{y}} \frac{\omega_{i, y}}{c} \frac{N_{i}}{\lambda_{e}} \frac{1}{\sigma_{y}\left(\sigma_{y}+\sigma_{x}\right)} \\
& =N_{i}\left(\frac{r_{p}}{A \lambda_{e}}\right)^{1 / 2}\left[\frac{2}{k_{y}} \frac{1}{\sigma_{y}\left(\sigma_{y}+\sigma_{x}\right)}\right]^{3 / 2},
\end{aligned}
$$

where $\lambda_{e}=N_{e} / S_{b}$ is the line density of electron bunch and $S_{b}$ is bunch spacing. The parameter $k_{x, y}=\sum_{x, y}\left(\sum_{x}+\sum_{y}\right) /$ $\left[\sigma_{x, y}\left(\sigma_{y}+\sigma_{x}\right)\right]$ is derived from the integration over two Gaussian distributions, representing the beam and ion cloud. As shown in Sec. II, the transverse distribution of ions is not Gaussian, but the electric field of the ion cloud closely approximates that of a Gaussian distribution with $\sigma_{i}^{2} \approx \sigma_{e}^{2} / 2$. Therefore, $k_{x}=k_{y} \approx 3 / 2$ with this approximation. For a flat beam, the vertical wake is stronger than the horizontal one. Therefore, there is a stronger instability in the vertical plane. We will discuss the instability in the vertical direction only in the rest of this paper and omit the subscript $y$ in some variables for simplicity.
Note that the wake of ions given by [14] differs from our result shown in Eq. (10) by a factor of 4/3. The ion frequency in [14] is also different from ours and others $[6,7]$ by a factor of $2 / 3$. Our model is more accurate and has good agreement with simulation as shown later.

Because of the nonlinear space charge force between electron beam and ion cloud, the wake of the ion cloud has a finite quality factor $Q$ which characterizes the frequency spread of the individual ions. Therefore, more precisely, the wake of the ion cloud has the following form:

$$
W(z)=\hat{W} e^{-\left(\omega_{i} z / 2 Q c\right)} \sin \left(\frac{\omega_{i} z}{c}\right) .
$$

Figure 4 shows the simulated wake of the ion cloud for various offsets $\Delta y_{e 0}$ of the test bunch. The test bunch initially has a vertical offset $\Delta y_{e 0}$ (for the vertical wake calculation) and the following bunches have zero offset. The ion cloud is disturbed by the test bunch due to the offset and then starts an oscillation. The oscillated ion cloud gives a vertical kicker to the following bunches $\Delta y_{e}^{\prime}$. The wake is calculated as $W_{y}(z)=$ $\gamma \Delta y_{e}^{\prime}(z) /\left(N_{e} r_{e} \Delta y_{e 0}\right)$. Here $z$ is the distance between the test bunch and the bunch which receives the kicker from the ion cloud. The wake is insensitive to $\Delta y_{e 0}$ when $\Delta y_{e 0}$ is smaller than the beam size, which means the electric field of the ion cloud has good linearity. However, when the bunch's offset is larger than the beam size, the wake becomes smaller and has strong nonlinearity due to the nonlinear field shown in Fig. 3. Therefore, the above wake model works well only in the linear regime, where the fast instability occurs as shown by simulations in the following section; hence, the model of the wake can still give the most important information about the beam-ion instability.

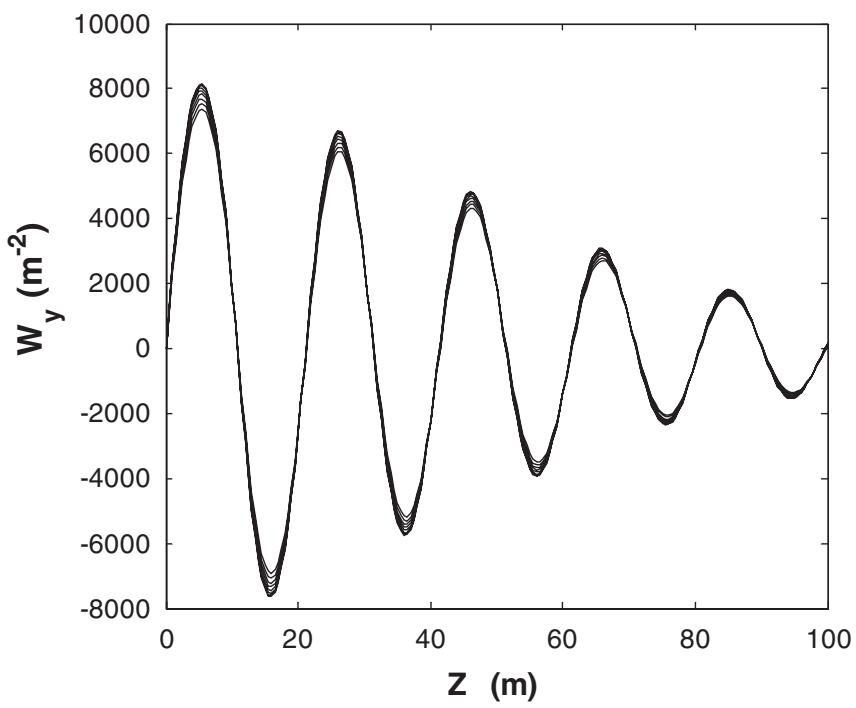

FIG. 4. Simulated wake of ion cloud for various $\Delta y_{e 0}$ ranging from $0.1 \sigma$ to $1 \sigma$ with a step of $0.1 \sigma$. The beam parameters are $\sigma_{x}=1.19 \mathrm{~mm}, \sigma_{y}=7.56 \mu \mathrm{m}, N_{e}=5 \times 10^{9}, S_{b}=0.5 \mathrm{~m}$, and $A=28\left(\mathrm{CO}^{+}\right)$. 
The wake due to electron cloud in a magnetic field free region is short range wake and can cause head-tail instability [17]. However, the wake of ion cloud is long range wake due to the heavier mass of ions and therefore it may drive coupled bunch instability. For instance, the frequency of the wake due to ion cloud in SPEAR3 ranges from a few of $\mathrm{MHz}$ to $70 \mathrm{MHz}$ in the $500 \mathrm{~mA}$ operation mode. The frequency depends on the ion species and also varies along the ring due to the variation of the beam size. The wavelength of the wake can be as large as $30.0 \mathrm{~m}(10 \mathrm{MHz})$ while the bunch spacing is only $0.63 \mathrm{~m}$.

Figure 5 shows the simulated wakefield for different bunch spacing with a constant beam line density $\lambda_{e}$. For instance, the bunch intensity doubles when the bunch spacing is doubled. The number of ions is the same in all cases. It clearly shows that the wakefield does not depend on the bunch spacing; instead, it depends on the beam line density. This agrees well with the analytical model.

Figure 6 compares the wakes by numerical and analytical approaches. A $Q$ of 9 is assumed for the analytical method. There is a good agreement in both frequency and amplitude. Note that the analytical approach used an approximation model of the ion's distribution and dimension. The simulated wake can also be fitted using the form of Eq. (11) to get the amplitude, frequency, and $Q$. For example, the fitted parameters of the simulated wake in Fig. 5 are $\hat{W}=9827 \mathrm{~m}^{-2}, Q=9$, and $\omega_{i}=92 \mathrm{MHz}$. The analytical formula gives $\hat{W}=9671 \mathrm{~m}^{-2}$ and $\omega_{i}=$ 84.95 MHz. The frequency and amplitude from analytical approach are $8 \%$ and $16 \%$ smaller than the simulated ones, respectively. This is fundamentally consistent with the field comparison shown in Fig. 3. A previous analysis result gives a $Q$ of 16 [7], which is larger than the numerical one. Figure 7 shows the dependence of the amplitude and frequency of the wake on the beam line density by

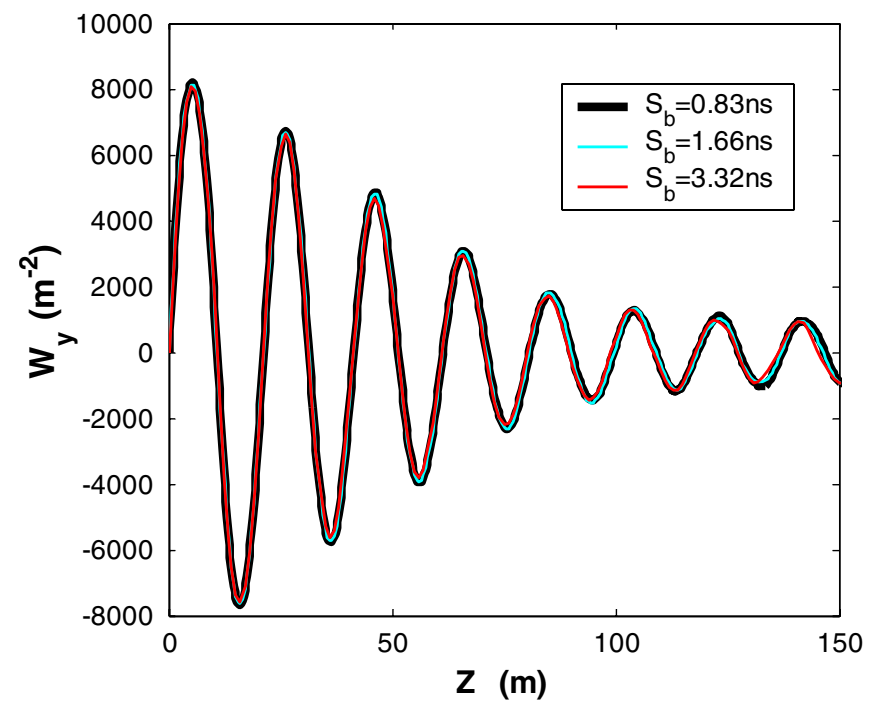

FIG. 5. Wake of ion cloud for various bunch spacing with a constant beam line density and number of ions.

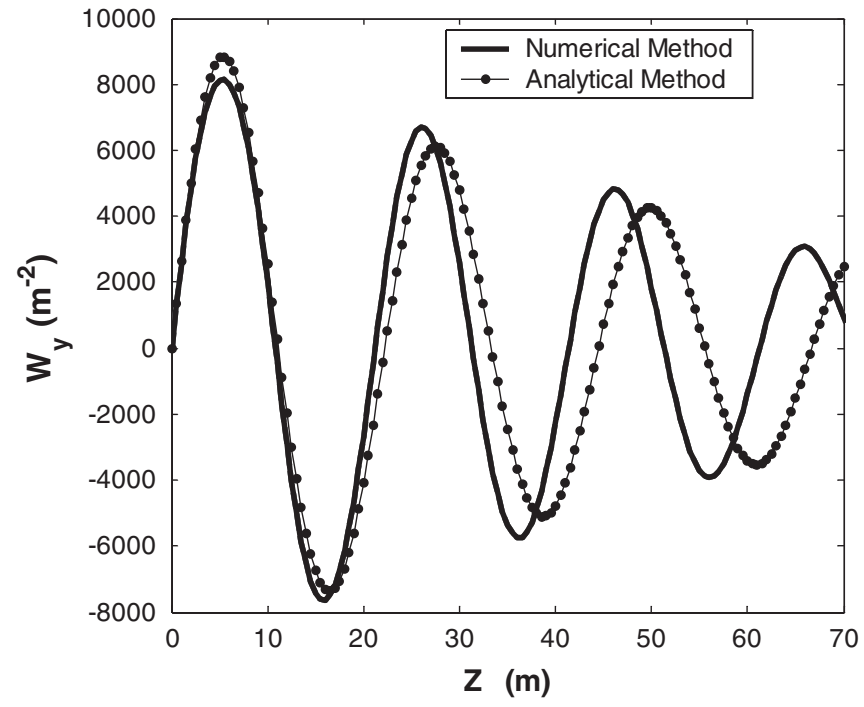

FIG. 6. Comparison of the wake due to ion cloud given by numerical and analytical methods, $\Delta y_{e 0}=0.13 \sigma$ is used in the simulation and $Q=9$ is used in the analysis.

simulation. There is a single bunch train in the beam. The amplitude, frequency, and $Q$ are found by fitting the wake with the model in Eq. (11). Simulation shows that the $Q$ does not change with beam line density. As shown in the figure, both amplitude and frequency linearly increase with the square root of the beam line density.

In summary, the wakefield of an ion cloud has the following characteristics: (i) When the wavelength of ion oscillation is much longer than the bunch spacing, the wake (both frequency and amplitude) depends on the average beam line density $\lambda_{e}$, instead of the bunch spacing or the bunch current. In other words, the wake depends on the average force of the electron beam because the ions move

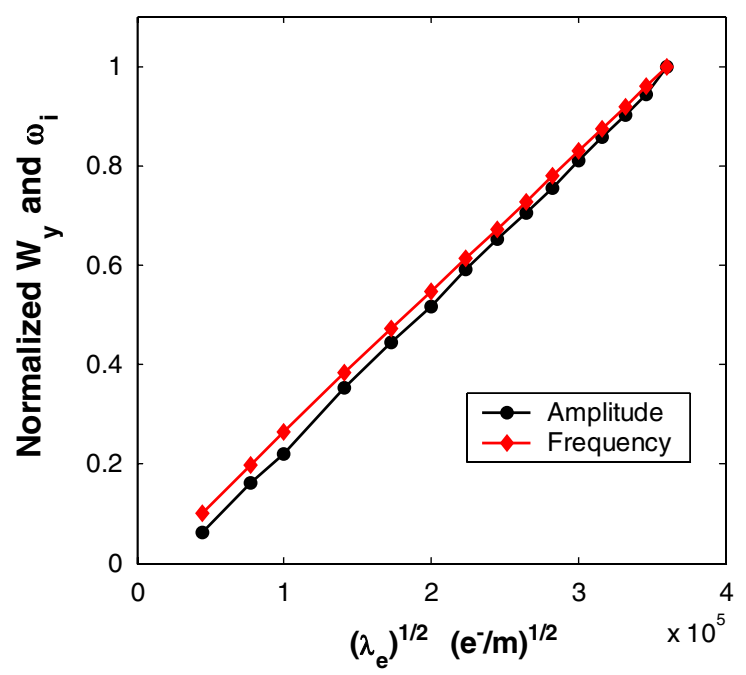

FIG. 7. Dependence of the amplitude and frequency of the wake on the beam line density by simulation. Both the amplitude and frequency are normalized in order to clearly display both of them in one plot. The beam filling pattern is single bunch train. 
slowly. (ii) Its frequency is the same as the ion's oscillation frequency, which is proportional to the square root of the beam line density as shown in Fig. 7. (iii) The amplitude of the wake is proportional to the line density of ion cloud and inversely proportional to the square root of the beam line density. For a single bunch-train filling pattern, the ion density is proportional to the beam density. Therefore, the wake is ultimately proportional to the square root of the beam line density: $\hat{W} \propto \lambda_{e}^{1 / 2}$ as shown in Fig. 7. (iv) For a flat beam $\left(\sigma_{x} \gg \sigma_{y}\right), \hat{W} \propto \sigma_{x}^{-3 / 2} \sigma_{y}^{-3 / 2}$. Therefore, there is a strong wake for a small beam. (v) The model of wake works only in the linear region as expected. When the amplitude of a bunch's oscillation is larger than the beam size, the force received by the electron bunches has strong nonlinearity, which slows down the instability as shown late by simulation. Since the fast instability occurs in the linear regime, the wake model is very useful to estimate the fast growth rate. (vi) The nonlinearity of the ion cloud gives a small $Q$ which is normally less than 10 .

With an assumption of a constant ion line density and beam size along the ring, the total wakefield due to the ion cloud in the whole ring can be written as

$$
W_{y, \text { total }}(z)=\hat{W}_{t} e^{-\left(\omega_{i} z / 2 Q c\right)} \sin \left(\frac{\omega_{i} z}{c}\right),
$$

with the amplitude of the wake

$$
\hat{W}_{t}=\frac{2 \omega_{i}}{c} \frac{L}{\lambda_{e}} \rho_{i, \mathrm{eff}},
$$

where $L$ is the circumference of the ring and $\rho_{i, \text { eff }}$ is the effective ion density, which is defined as

$$
\rho_{i, \mathrm{eff}}=\frac{\lambda_{i}}{k_{y} \sigma_{y}\left(\sigma_{y}+\sigma_{x}\right)} .
$$

There is a flat beam for most electron rings with $\sigma_{x} \gg \sigma_{y}$. The factor of $1 / k_{y}$ in the effective ion density represents the dependence of wake on the ion's distribution.

In the above discussion, a constant beam size is used to estimate the total wake of the ring, which should be calculated by integration along the ring to include the variation of the beam size. Moreover, the motions of ions in both horizontal and vertical directions are assumed to be stable. If the ion's motion is unstable, the ion density becomes smaller.

The Fourier transformation of the wake gives the impedance in the frequency domain as

$$
Z_{\text {ion }}(\omega) \approx \frac{\hat{W}_{t}}{\omega} \frac{Q}{1+i Q\left(\frac{\omega_{i}}{\omega}-\frac{\omega}{\omega_{i}}\right)} .
$$

When the beam is evenly filled along the ring, the exponential growth rate of the coupled bunch instability for mode $y_{j}^{\mu} \propto e^{2 \pi \mu j / n_{b}}$ is [18]

$$
\frac{1}{\tau^{\mu}}=\frac{N_{e} n_{b} r_{e} c}{2 \gamma T_{0}^{2} \omega_{\beta}} \sum_{p=-\infty}^{\infty} \operatorname{Re}\left\{Z_{\text {ion }}\left[\left(p n_{b}+\nu_{y}+\mu\right) \omega_{0}\right]\right\} .
$$

Here $\nu_{y}$ and $\omega_{\beta}$ are the betatron tune and frequency, $T_{0}$ and $\omega_{0}$ are revolution period and frequency. At the resonance, $\omega=\omega_{i}$, the real part of the effective impedance can be simplified to

$\operatorname{Re}\left\{\sum_{p=-\infty}^{\infty} Z_{\text {ion }}\left[\left(p n_{b}+\nu_{y}+\mu\right) \omega_{0}\right]\right\} \approx \frac{\omega_{i}}{\omega} R=\frac{\hat{W}_{t}}{\omega_{i}} Q$.

This gives a maximum growth rate

$$
\frac{1}{\tau} \approx \frac{r_{e} c \beta_{y} \rho_{i, \mathrm{eff}} Q}{\gamma}=\frac{r_{e} c \beta_{y} Q}{\gamma} \frac{\lambda_{i}}{k_{y} \sigma_{y}\left(\sigma_{y}+\sigma_{x}\right)} .
$$

The relationships $\omega_{\beta} T=L / \beta_{y}$ and $L \lambda_{e}=L N_{e} / S_{b}=$ $N_{e} n_{b}$ are used in the above derivation.

The frequency of the wake depends on the transverse beam size. Accordingly, the variation of the betatron function along the storage ring induces a frequency spread. This frequency spread results in a reduction of the effective $Q$ of the wake. For instance, the exponential growth rate of the fast ion beam instability of a single bunch-train beam with a frequency variation of $\Delta \omega_{i}$ due to the variation of beam size is [8]

$$
\frac{1}{\tau_{e, \mathrm{FII}}} \approx \frac{r_{e} c \beta_{y} \lambda_{i}}{3 \sqrt{2} \gamma \sigma_{y}\left(\sigma_{y}+\sigma_{x}\right)} \frac{1}{\Delta \omega_{i} / \omega_{i}} .
$$

Here $\lambda_{i}$ is the ion line density at the end of the bunch train. The frequency variation of $\Delta \omega_{i}$ has a similar Landau damping mechanism as the nonlinear space charge effect in Eq. (18). If we define an effective $Q$ due to this frequency spread as

$$
Q_{\text {optics }}=\frac{\omega_{i}}{\sqrt{2} \Delta \omega_{i}},
$$

for example, an optics with $\Delta \omega_{i} / \omega_{i}=0.2$ gives a $Q_{\text {optics }}$ of 3.54. Then Eq. (19) can be rewritten as

$$
\frac{1}{\tau_{e, \mathrm{FII}}} \approx \frac{1}{2} \frac{r_{e} c \beta_{y} \rho_{i, \mathrm{eff}} Q_{\mathrm{optics}}}{\gamma} .
$$

The two types of instability represented in Eqs. (18) and (21) have the same form except a factor of $1 / 2$ in the FII case which gives the average density along the bunch train. Note that the ion density linearly increases along the bunch train for the single bunch-train case. If we use the average ion density over the bunch train, the two equations will be identical: the growth rate is proportional to the average effective ion density near the beam

$$
\frac{1}{\tau} \approx \frac{r_{e} c \beta_{y} \bar{\rho}_{i, \mathrm{eff}} Q}{\gamma} .
$$

Roughly speaking, the frequency spread due to the optics and the nonlinearity of ion cloud has the equivalent effect in damping the instability. $Q$ in the above equation can include both effects.

There are multigas species in the beam's vacuum chamber. The dominant species are hydrogen, methane, water, carbon monoxide, and carbon dioxide in most electron 
rings. Each type of ion has its own frequency and frequency spread due to the variation of beam size along the ring. Therefore, additional frequency spread may be added when multispecies are considered. This effect is mixed with the trapping condition, bunch-train gap effect, and optics effect. Simulation shows that the additional damping effect due to multigas species is small [19]. Another simulation concludes that the superposition rule applies for multispecies of ions [9]. In this case, one can independently calculate the growth rate due to individual gas species. Note that the growth rate does not depend on the ion frequency, but the unstable mode number does. The superposition rule may fail for some rings. Detail parameters need to be checked for each case. For most electron rings, the frequency of hydrogen is well separated from the frequencies of other types of ions. As a result, hydrogen does not contribute to additional frequency spread. The frequencies of carbon monoxide and carbon dioxide are close to each other. They may overlap due to the variation of the beam size along the ring and result in an increment of the total frequency variation.

In the above analysis the ion motion within the bunch train is assumed to be stable, namely $\omega_{i} S_{b} / c \ll 1$. In the linear theory, the motion of ion is stable if its mass is larger than the critical mass number:

$$
A_{x, y}=\frac{N_{e} r_{p} S_{b}}{2\left(\sigma_{x}+\sigma_{y}\right) \sigma_{x, y}} .
$$

For example, most ions are stable in SPEAR3. However, the light ions, such as the hydrogen ion, can become unstable for an ultrasmall emittance beam, for instance, PEPX beam [20]. The strong beam force overfocuses the ions and makes the motion of ions within the single bunch train unstable. Therefore, there is a self-mitigation of the beam-ion instability for ultrahigh intensity beam. Analysis of the beam-ion instability with ultrahigh intensity beam is not feasible and a simulation with realistic vacuum is required. In general, the beam-ion instability can be divided into following four categories: (i) conventional ion trapping instability: the motion of ion over one turn is stable, therefore, the ions are trapped for multiple revolutions; (ii) fast beam-ion instability: the motion of ion along single bunch train is stable and there is a long bunch-train gap so that all ions are cleared out after the gap; (iii) untrapped beam-ion instability: the motion of ion along single bunch train is unstable (this occurs for very high intensity beam); (iv) multibunch train instability: combination of the above three types of instabilities.

\section{MITIGATION OF THE BEAM INSTABILITY WITH MULTIBUNCH TRAIN BEAM FILLING}

Table I compares the main parameters and the driving force of the beam-ion instability in several existing and designed electron rings. The emittance of the future rings is up to 2 orders of magnitude smaller than the present B-factory. Note that a constant beam size is used for each ring and the trapping condition is not applied. The last column shows the normalized $\rho_{i, \text { eff }} / \gamma$ with the assumption of a single bunch-train beam filling pattern. The value of $\rho_{i, \text { eff }} / \gamma$ in KEKB is assumed to be 1 . With the single bunch-train beam, the ion density is proportional to the total number of electrons in the ring. Among the listed existing rings, SSRF, KEK ATF, SPEAR3, and SLS have relatively strong instability due to their small beam size. The beam-ion instability has been observed in PLS [21], SOLEIL [22], SSRF [23], and SPEAR3. Vertical emittance

TABLE I. Comparison of main parameters and driving force of beam-ion instability in existing rings and future rings (italic). Single bunch beam filling pattern and constant vacuum pressure are assumed in the comparisons. The average betatron function is used in the estimation of the beam size.

\begin{tabular}{llcrrccrc}
\hline \hline Ring & $E(\mathrm{GeV})$ & Cir $(\mathrm{m})$ & \multicolumn{1}{c}{$n_{b}$} & $I(\mathrm{~mA})$ & $L_{\mathrm{SP}}(\mathrm{m})$ & $\epsilon_{x} / \sigma_{x} \mathrm{~nm} \cdot \mathrm{rad} / \mu \mathrm{m}$ & $\epsilon_{y} / \sigma_{Y} \mathrm{~nm} \cdot \mathrm{rad} / \mu \mathrm{m}$ & $\rho_{i, \text { eff }} / \gamma$ \\
\hline PEPII & 8.0 & 2199 & 1588 & 1550 & 1.26 & $31 / 660$ & $1.4 / 144$ & 1.1 \\
KEKB & 8.0 & 3016 & 1387 & 1200 & 2.1 & $24 / 500$ & $0.49 / 75$ & 1.0 \\
ALS & $1.5 / 1.9$ & 196.8 & 320 & 400 & 0.6 & $6.3 / 160$ & $0.06 / 23$ & 1.2 \\
APS & 7.0 & 1104 & 24 & 100 & 45.9 & $3.0 / 276$ & $0.025 / 11$ & 0.4 \\
PLS & 2.0 & 280 & 180 & 360 & 0.6 & $12.1 / 350$ & $0.12 / 35$ & 0.3 \\
AS & 3.0 & 216 & 300 & 200 & 0.6 & $10.3 / 165$ & $0.03 / 14$ & 0.5 \\
SSRF & 3.5 & 432 & 450 & 200 & 0.6 & $3.9 / 110$ & $0.0273 / 13$ & 1.4 \\
KEK ATF & 1.3 & 138 & 20 & 64 & 0.77 & $1.2 / 70$ & $0.0045 / 5$ & 1.6 \\
SPEAR3 & 3.0 & 234 & 280 & 500 & 0.63 & $10 / 200$ & $0.014 / 10$ & 1.6 \\
SLS & 2.4 & 288 & 390 & 400 & 0.6 & $5.5 / 111$ & $0.005 / 5.6$ & 6.4 \\
NSLSII & 3.0 & 792 & 1040 & 500 & 0.6 & $1.0 / 122$ & $0.008 / 11$ & 8.1 \\
SuperKEKB I & 3.5 & 3016 & 5120 & 9400 & 0.6 & $24 / 600$ & $0.24 / 60$ & 18.7 \\
SuperKEKB II & 7.0 & 3016 & 2500 & 2600 & 1.18 & $5.1 / 280$ & $0.013 / 14$ & 23.7 \\
ILC & 5.0 & 6000 & 3000 & 400 & 0.5 & $0.8 / 120$ & $0.002 / 6$ & 55.3 \\
PEPX & 4.5 & 2199 & 3154 & 1500 & 0.6 & $0.08 / 18$ & $0.0043 / 5.7$ & 596 \\
\hline \hline
\end{tabular}


blowup has been observed in Australian Synchrotron (AS) [24], which could be an indication of the beam-ion instability. Beam-ion instability has not been reported in SLS with a normal operation filling pattern. But it is expected according to our first order estimation listed in Table I. Two options for SuperKEKB are listed: the high charge option (SuperKEKB I) and low charge with low emittance option (SuperKEKB II). The low charge option has stronger instability due to its small emittance. The ultrasmall emittance in the PEPX can drive the strongest beam instability. The beam-ion instability is not a problem for most of the existing electron rings. However, it can be a serious problem for the future rings with high beam current and small emittance as shown in Table I.

In order to mitigate the instability, one direct solution is to reduce the ion density near the beam. A low ion density can be achieved using a clearing electrode, a better vacuum, or beam shaking. Here we study one simple and effective way to reduce ion density by using a multibunch train beam filling pattern. The instability with a gap is studied in $[25,26]$.

\section{A. Diffusion of ion cloud during the train gap}

We begin with the ion's single particle motion under the potential of electron beam. In the linear regime, the Hamiltonian for an ion is

$$
H\left(x, p_{x}, y, p_{y}\right)=\frac{p_{x}^{2}}{2 m_{i}}+\frac{p_{y}^{2}}{2 m_{i}}+\frac{1}{2} m_{i} \omega_{i, x}^{2} x^{2}+\frac{1}{2} m_{i} \omega_{i, y}^{2} y^{2} .
$$

Here $m_{i}$ is the mass of ion. The ions initially have the same distribution as the electrons. A good approximation is obtained by assuming that the ions are born at zero velocity since the beam's potential is much larger than the ions' thermal energy. Therefore, the displacement and velocity of the ion in the vertical direction follow

$$
\begin{gathered}
y(t)=y_{0} \sin \left(\omega_{i} t+\phi_{0}\right), \\
v(t)=y_{0} \omega_{i} \cos \left(\omega_{i} t+\phi_{0}\right),
\end{gathered}
$$

where $y_{0}$ is the ion's oscillation amplitude, which is approximately the position at its birth. $\varphi_{0}$ is the ion's initial phase when the ions are born, which has a uniform distribution due to the random process of ionization. Hence, the ion's speed has the following distribution:

$$
f(\xi)=\frac{2}{\pi} \frac{1}{\sqrt{1-\xi^{2}}}
$$

where $\xi=v / \omega_{i} y_{0}$. The distribution is shown in Fig. 8. It is seen from this plot that the distribution has a sharp peak at the maximum speed. During the bunch-train gap, the ions drift with constant velocity (ions' self-space charge effect is neglected). The speed distribution in Eq. (27) indicates that the ion density near the beam decay fast at the beginning and

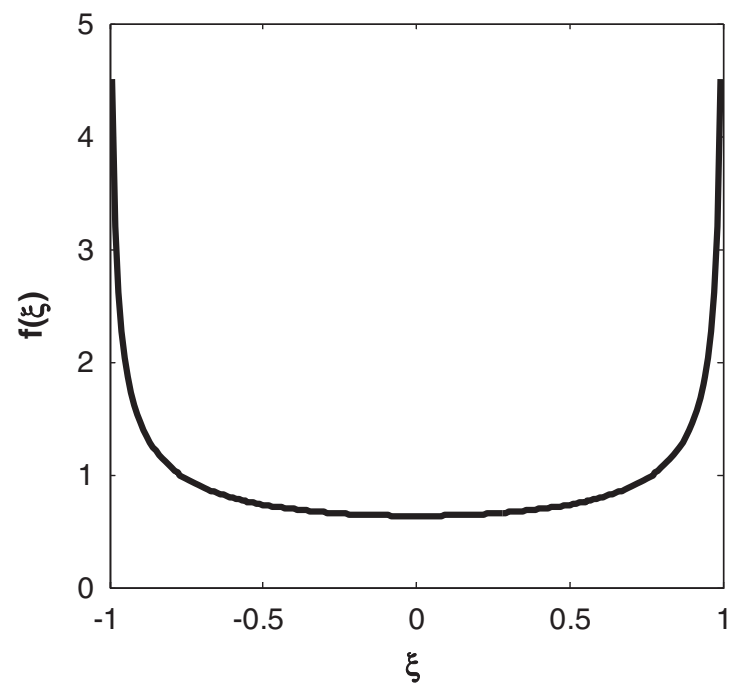

FIG. 8. Distributions of the speed of ion particles.

then slows down. Figure 9 shows the simulated buildup of ions near the beam and its decay during the train gap. The ion density linearly increases along the bunch train. During the train gap it is roughly an exponential decay with a decay time order of the ion's oscillation period. This can be explained by Eq. (26), which shows ions with a short oscillation period drift quickly during the gap. The experiment in SPring-8 also shows a similar exponential decay of the ion density, beam size, and betatron tune shift during the train gap [27].

The linear motion of ions with a bunch-train gap can also be studied using a transportation matrix. The ion receives a linear kicker during the passage of an electron bunch (acting like a focusing quadrupole magnet) and then drifts during

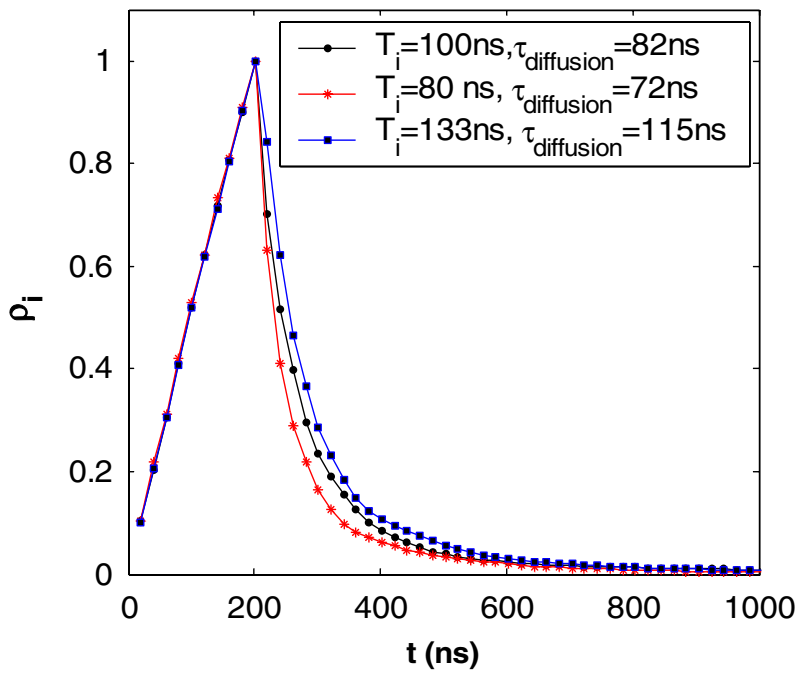

FIG. 9. Buildup of ion cloud along a short bunch train and its decay during the train gap for various ions' oscillation frequencies. The ion density is normalized so that the peak density at the end of the bunch train is 1 . The calculated ion oscillation period $T_{i}$ and the simulated ion decay time $\tau_{\text {diffusion }}$ during the bunchtrain gap are also shown in the plot. 


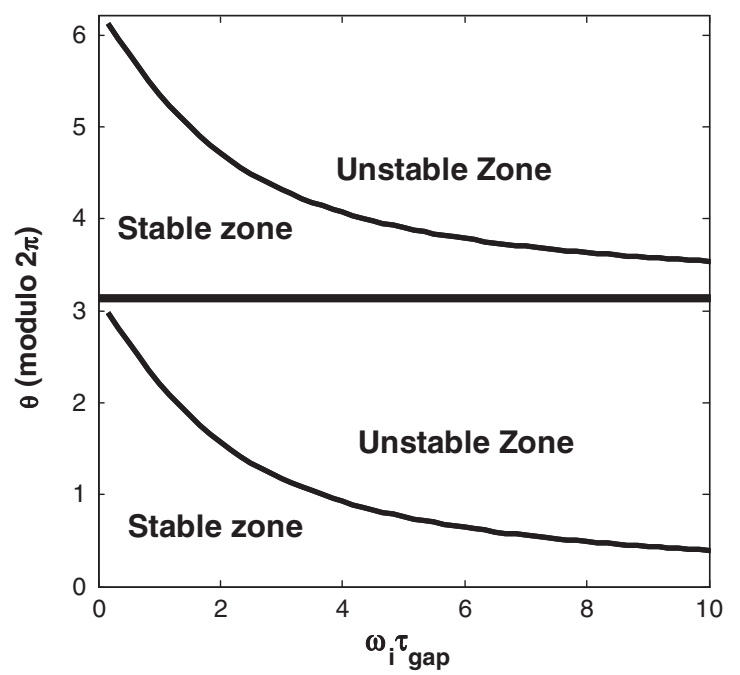

FIG. 10. Variation of stable and unstable zones with the length of the bunch-train gap.

the bunch-spacing time. The bunch-train gap can be represented by a long drift period. Therefore, we can obtain the one turn transportation matrix of the ion motion. When a gap $\tau_{\text {gap }}$ is applied with a bunch-train length $\tau_{\text {train }}$, the stable condition of the ion's linear motion becomes [28]

$$
n \pi<\theta<n \pi+2 \operatorname{arctg} \frac{2}{\omega_{i} \tau_{\text {gap }}},
$$

where $\theta=\omega_{i} \tau_{\text {train }}$. With the increment of the gap, the unstable region increases as shown in Fig. 10. A gap of one period of ion oscillation already provides a large unstable zone (more than 60\%). The unstable zone increases dramatically with a gap of 1-2 ion oscillation periods and then slows down with further increment of the bunch-train gap. This is consistent with the exponential decay of the ion cloud given by simulation and experiment.

The minimum required train gap is a few of the ion's oscillation periods. There is a small ion's oscillation period for a high beam current with a small emittance. Therefore, a shorter train gap is needed to clear the ions for higher intensity beams. In other words, the bunch-train gap is more effective for high intensity beam with small emittance.

\section{B. Reduction of ion density by using multibunch train beam filling}

The exponential decay makes it possible to effectively reduce the trapped ions by using a multibunch train beam filling pattern with a short train gap. This works especially well for high intensity beams. We assume there are $N_{\text {train }}$ bunch trains evenly distributed along the ring. Each bunch train consists of $M$ bunches and is followed by a bunchtrain gap $\tau_{\text {gap }}$. The density of ion cloud during the bunchtrain gap exponentially decays with a decay time of $\tau_{\text {diffusion, }}$, which is of the order of the ion oscillation period.
Therefore, the ion density after the passage of $n$th bunch train becomes

$$
\rho_{n}=g M N_{e} \sum_{k=0}^{n-1} \exp \left(-k \tau_{\text {gap }} / \tau_{\text {diffusion }}\right) .
$$

Here $g$ is a constant parameter for given vacuum pressure and beam size

$$
g=\delta_{i} \frac{P}{k T} \frac{1}{k_{y} \sigma_{y}\left(\sigma_{x}+\sigma_{y}\right)} .
$$

After a long enough period of time, the ion density reaches saturation level at

$$
\rho_{\infty}=g M N_{e} \frac{1}{1-e^{-\tau_{\text {gap }} / \tau_{\text {diffusion }}}} .
$$

With this scheme, the density of trapped ions can be significantly reduced compared to a long single train filling pattern by a factor

$$
\begin{aligned}
& f_{\text {gap }}=\frac{\rho_{\infty}}{g M N_{\text {train }} N_{e}}=\frac{1}{N_{\text {train }}} \frac{1}{1-e^{-\tau_{\text {gap }} / \tau_{\text {diffusion }}},} \\
& \left(N_{\text {train }} \ll n_{b}\right) .
\end{aligned}
$$

It can be concluded from the above equation that the multibunch train filling is an effective way to reduce the ion density in a large storage ring (therefore a large $N_{\text {train }}$ ) with a low emittance (short $\tau_{\text {diffusion }}$ ). In electron rings, the vertical emittance is smaller than the horizontal emittance and therefore the vertical motion of the ions with gaps becomes unstable first. Strictly speaking, Eq. (32) is valid for beams with large aspect ratios where the short gaps destabilize only the vertical ion trapping. One can multiply the left side of Eq. (32) by a similar factor due to the horizontal decay of the ions if the gap length is not negligible compared to the ion's horizontal oscillation period.

PEPX is a proposed light source using the existing PEPII $2.2 \mathrm{~km}$ tunnel in SLAC. Figure 11 shows the buildup of ion cloud with 183 bunch trains. The ion density near the beam saturates after three bunch trains and it is reduced by a factor of 130 compared to the single bunch-train filling pattern. The beam-ion instability growth rate depends linearly on the density of ion cloud. Therefore, there is a significant reduction of the instability growth rate by applying the multibunch train filling pattern.

For a given circumference of the accelerator ring, the normalized ion density $f_{\text {gap }}$ can be minimized by choosing different $N_{\text {train }}$ and $\tau_{\text {gap }}$. The saturated ion density after a long enough of period of time can be found for each beam filling pattern by simulation as Fig. 11 shows. In this way, we can get the saturated ion-density dependence on the beam filling pattern by simulation. NSLSII is a new light source under construction in Brookhaven National Laboratory in the United States. It is a $792 \mathrm{~m}$ storage ring with beam energy of $3 \mathrm{GeV}$. The main parameters are listed in Table I. Figure 12 shows the variation of ion 


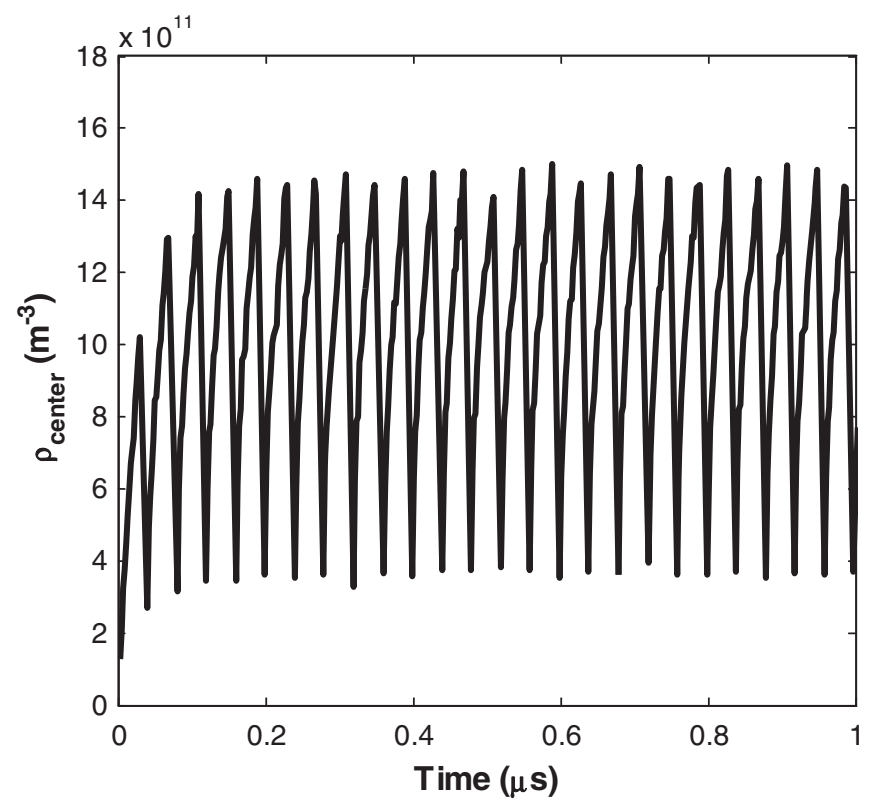

FIG. 11. Buildup of ion cloud in PEPX. The pressure is 0.3 nTorr $\left(\mathrm{CO}^{+}\right)$. The beam consists of 183 bunch trains. The horizontal emittance is $81 \mathrm{pm}$ and vertical emittance is $4.3 \mathrm{pm}$. The density near the beam is the ion density within $\sqrt{3} \sigma$.

density near the beam in the NSLSII with the number of bunch train by simulation and the calculated $f_{\text {gap }}$ from Eq. (32). The simulated ion density is normalized by the density of one long bunch-train filling pattern case.

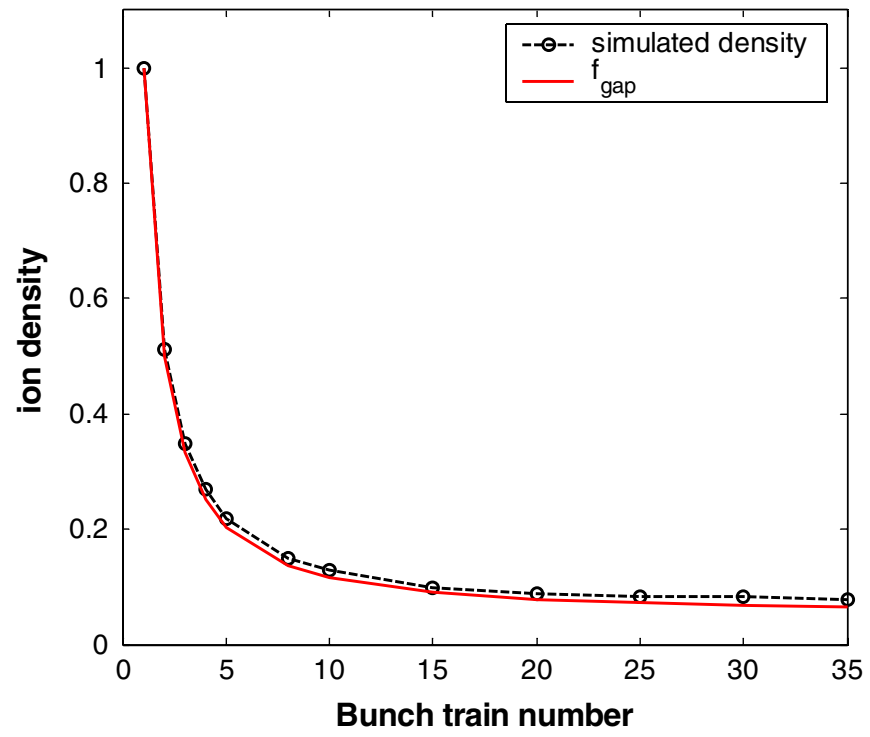

FIG. 12. Normalized ion-density near the beam for various numbers of bunch trains by simulation and analysis in NSLSII. The density is normalized by the density of one bunch-train filling pattern. The total number of bunches (1000), bunch current $(0.5 \mathrm{~mA})$, and bunch spacing $(2 \mathrm{~ns})$ are the same while the bunch-train gap is adjusted to keep the length of the ring constant. A diffusion time of $31.60 \mathrm{~ns}$, which is equal to the ion oscillation period calculated using the average betatron function along the whole ring, is used in the calculation of $f_{\text {gap }}$.
Therefore, the density has the same meaning as $f_{\text {gap }}$. The simulation agrees very well with the analysis. It shows that the ion density can be effectively reduced with 20 bunch trains. The reduction speed becomes slow with a further increment of the bunch-train number due to a shorter train gap.

A multibunch train filling pattern is a combination of uniform beam filling (with a constant ion density) and single bunch-train filling pattern where the ion density linearly increases along the bunch train. The instability growth rate is proportional to the average ion density seen by the beam. We define the ratio of the average ion density to the maximum ion density as

$$
a=\bar{\rho} / \rho_{\infty}, \quad(0.5 \leq a \leq 1) .
$$

Then the growth rate with a multibunch train filling pattern can be written as

$$
\frac{1}{\tau} \approx \frac{a r_{e} c \beta_{y} \rho_{\infty} Q}{\gamma}, \quad(0.5 \leq a \leq 1) .
$$

The above equation can be considered to be the instability growth rate for a general beam filling pattern with $a=1$ for an even beam filling pattern and $a=0.5$ for a single bunch-train beam filling pattern.

\section{Simulation of beam instability with multibunch train filling in NSLSII, PEPX, and SuperKEKB}

Simulation has a number of advantages in the study of the beam-ion instability: the nonlinearity of the ion cloud is automatically included; the effects of optics and bunchtrain gap with arbitrary beam filling pattern can be easily handled; a realistic vacuum model with multigas species is straightforward in simulation.

There are two simulation codes in SLAC. One is based on the so-called strong-strong model. Both the ions and high energy bunches are represented by many macroparticles. Therefore it can simulate both the dipole instability and beam emittance blowup. Another code is based on the weak-strong model. The ion cloud is represented by many macroparticles, but the high energy bunch is represented by slices with rigid transverse bunch profile (sheet beam model). A weak-strong code is used in this paper in order to speed up the simulation.

The ions are distributed along the whole ring according to the vacuum pressure. The optics of the ring with a MAD [29] type of format is read as an input of the code. New ions are generated in each element when electron bunches pass by. The ions generated by previous bunches will receive a kicker from the following bunches and the self-field of the ions. Meanwhile the electron bunch also receives a kicker from the ion cloud in each element. The electric potential of ion cloud and high energy beam satisfies the Poisson equation,

$$
\Delta \phi=-\rho / \varepsilon_{0},
$$


where $\varepsilon_{0}$ is the permittivity of the vacuum. This equation is solved using the finite element method (FEM). At each location, the ions are assumed to be uniform in longitudinal direction and Eq. (35) becomes two dimensional. The finite element equation of Eq. (35) is

$$
\begin{gathered}
\mathbf{A} \phi=\mathbf{B}, \\
A_{i j}^{e}=\iint_{S}\left(\frac{\partial N_{i}}{\partial x} \frac{\partial N_{j}}{\partial x}+\frac{\partial N_{i}}{\partial y} \frac{\partial N_{j}}{\partial y}\right) d x d y \\
B_{i}^{e}=\frac{Q_{i}}{\varepsilon_{0}},
\end{gathered}
$$

where $x$ and $y$ are the transverse coordinates. $Q_{i}$ is the charge at the node $i$. The stiffness matrix $A$ depends only on the mesh and $\mathbf{B}$ represents the source term. $N_{i}$ is called the shape function in FEM. The ions near the beam are effective on the beam dynamics. In order to speed up the computation, we only consider the ions within a certain area near the beam center, for instance, 100 times beam size, instead of the whole beam pipe. FEM allows irregular mesh. A dense mesh near the beam and a coarse mesh near the boundary are used to reduce the number of grids and improve the accuracy. The ions beyond that area are discarded in the simulation. Since only a small area of the beam pipe is selected, one should carefully set the potential on the boundary. The potential on the boundary is calculated from the charges within the domain where we are interested in using the particle to particle model [30]. The charge of the macroparticle is assigned to the mesh node according to the shape function $Q_{i}=N_{i} Q_{0}$, and we directly calculate the electric field at each particle position using the potential at the mesh nodes:

$$
\mathbf{E}=-\sum_{i}\left(\frac{\partial N_{i}}{\partial x} \phi_{i} \hat{x}+\frac{\partial N_{i}}{\partial y} \phi_{i} \hat{y}\right) .
$$

The electric field of ion cloud shown in Fig. 3 is one example of the field by the FEM solver.

Since the transverse cross section of the high energy bunches does not change in the weak-strong model, the field of high energy bunches can be simply calculated analytically, for instance, using Bassetti-Erskine formal [31] (as shown in the Appendix) for bi-Gaussian beam profile. The electric field of a round Gaussian beam is

$$
E_{r}(r)=\frac{e N_{e}}{4 \pi \varepsilon_{0}} \frac{2}{r}\left[1-\exp \left(-\frac{r^{2}}{2 \sigma_{r}^{2}}\right)\right]
$$

However, it has to be calculated numerically in the strongstrong model because the transverse distribution of high energy beam varies with time.

The electron bunches are tracked through the accelerator element by element, and therefore, the modulation of the ion oscillation frequency due to the variation of the beam size is automatically taken into account. One may assume that ions are generated along part of the ring according to the symmetry of the optics, such as a half or a quarter of the ring, to speed up the calculation.

Multigas species, for instance, $\mathrm{H}_{2}, \mathrm{CH}_{4}, \mathrm{H}_{2} \mathrm{O}, \mathrm{CO}$, and $\mathrm{CO}_{2}$, are used in the code to represent the realistic vacuum pressure. Different types of ions are generated in each element according to the partial pressure and ionization cross section of the gas species.

Three examples are given in this section. The first one is the NSLSII. Figure 13 shows the simulated beam's vertical instability in NSLSII for different bunch-train numbers with a total bunch number of 1000 . A partial vacuum pressure of $1 \mathrm{nTorr}\left(\mathrm{CO}^{+}\right)$is used. It clearly shows the mitigation of the beam instability by using multibunch train beam filling as predicted in Fig. 12. The growth time with 20 bunch trains is 16 times longer than that of single bunch-train case.

Figure 14 shows the detail of beam instability with 20 bunch trains in NSLSII. There is minor improvement of the instability with further increase of the number of bunch trains because the train gap becomes shorter with an increasing number of bunch trains. The 20 bunch-trains filling pattern is close to the optimized one. The beam amplitude grows exponentially when the amplitude is small compared to the beam size, and then the growth is slowed down by the nonlinearity at large amplitude. The exponential growth time is about $1 \mathrm{~ms}$ for a vacuum pressure 1 nTorr of $\mathrm{CO}^{+}$, which is shorter than the radiation damping time of $13 \mathrm{~ms}$. A better vacuum, such as a pressure lower than 0.1 nTorr, or a feedback system is required to suppress the instability. Many unstable modes are excited due to the variation of the ion's frequency along

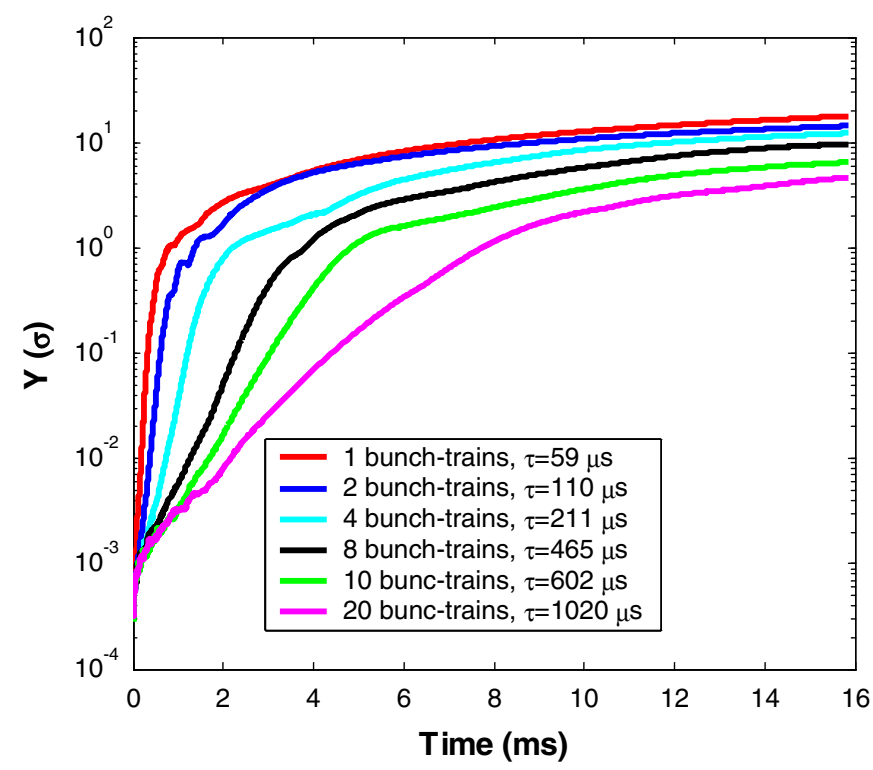

FIG. 13. Growth of the beam's vertical amplitude for various beam filling patterns in NSLSII. The amplitude is normalized by the vertical beam size. The total number of bunches is 1000 and the vacuum pressure is 1 nTorr of $\mathrm{CO}^{+}$. 

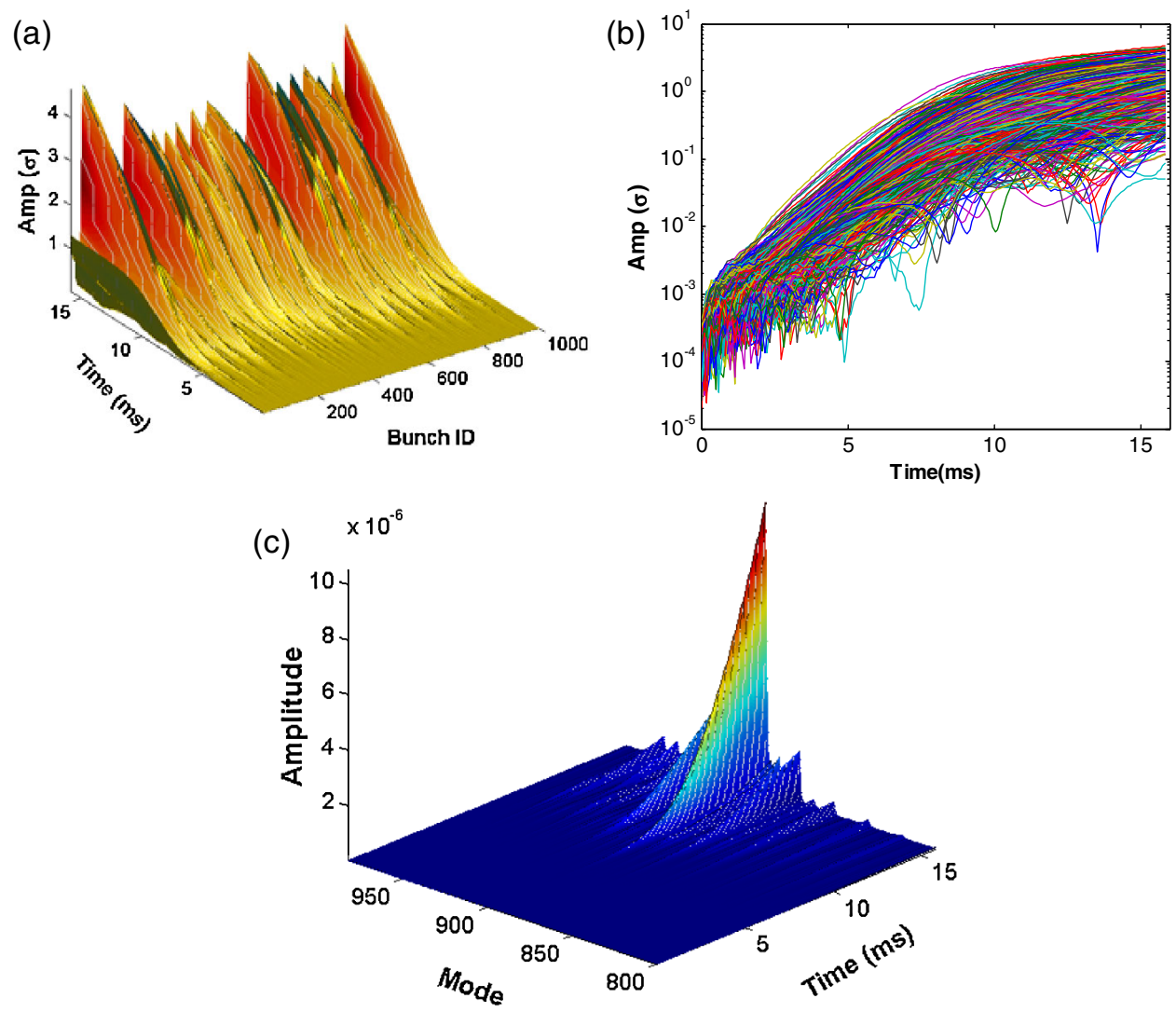

FIG. 14. Simulated vertical oscillation amplitude of different bunches (a), (b) and the unstable modes (c) in NSLSII. The amplitude is normalized by the beam size. The amplitude uses a linear scale in (a) to clearly show the oscillation amplitude of individual bunches and logarithmic scale in (b) to display the exponential growth. The exponential growth time is 1.0 ms. Different lines in (b) represent different bunches. There are 20 bunch trains. Each bunch train consists of 50 bunches followed by a bunch-train gap of $32 \mathrm{~ns}$, which is about one $\mathrm{CO}^{+}$oscillation period. The bunch spacing is $2 \mathrm{~ns}$ and bunch current is $0.5 \mathrm{~mA}$. The pressure is $1 \mathrm{nTorr}$ of $\mathrm{CO}^{+}$.

the ring even with single gas species $\mathrm{CO}^{+}$as shown in Fig. 14. Note that there can be multiple unstable modes even with a constant beam size because of the low $Q$ and high frequency of the wake:

$$
n_{\text {mode }}=\frac{\Delta \omega}{\omega_{0}} \approx \frac{\omega_{i}}{Q \omega_{0}} \gg 1 .
$$

The second example is PEPX. It is a proposed next general light source with ultrasmall natural emittance of $80 \mathrm{pm}$. The total number of bunches is up to 3154 . Figure 15 shows the simulated vertical beam-ion instability for a different beam filling pattern. A vacuum pressure of 0.3 nTorr $\mathrm{CO}^{+}$is assumed in the simulation. The growth rate of the instability is also given in the plot. Up to 183 bunch trains can be used to mitigate the beam-ion instability and the reduction of the instability growth rate is more than 2 orders of magnitude compared with the single bunch-train case. The multibunch train beam filling pattern is very effective in the reduction of the trapped ions in a large ring with a small emittance like PEPX. The growth time with 183 bunch trains is $0.67 \mathrm{~ms}$, which is much shorter

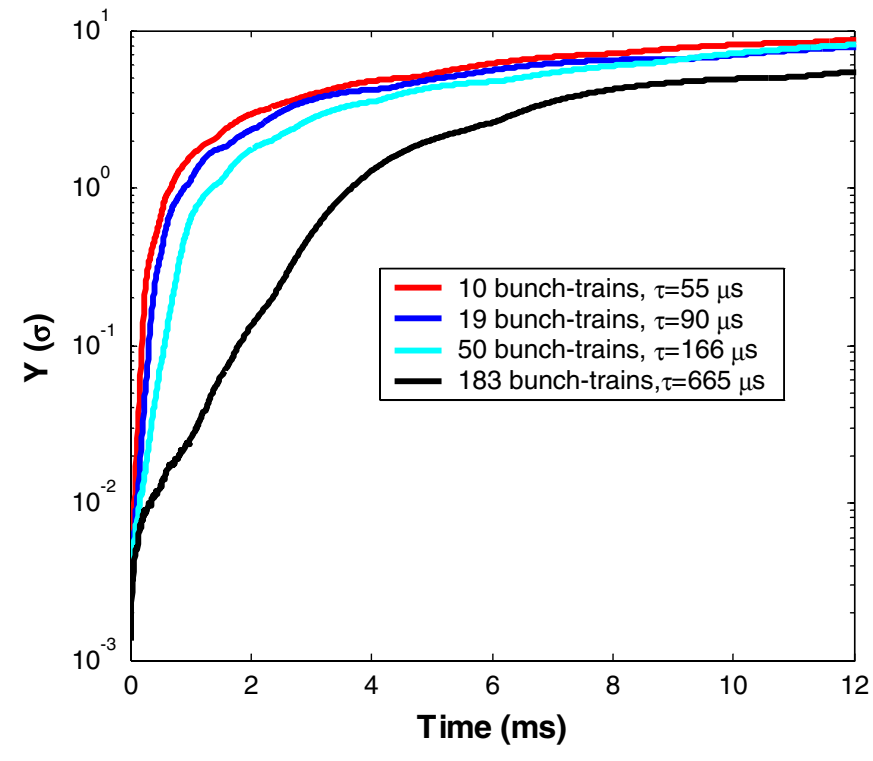

FIG. 15. Growth of the beam's vertical amplitude for various beam filling patterns in PEPX. The amplitude is normalized by vertical beam size. The vacuum pressure is 0.3 nTorr of $\mathrm{CO}^{+}$. 
than the radiation damping time of $21 \mathrm{~ms}$. Note a low pressure of 0.3 nTorr is used in the simulation. Apparently, a bunch-by-bunch feedback system is required.

The third example is SuperKEKB, which is the upgrade plan of the KEKB factory. Two options are listed in Table I. In this paper we estimate the instability of the high charge option only. Unlike KEKB, the electron beam may be stored in LER at SuperKEKB in order to mitigate the electron cloud effect. In this case, the beam energy is decreased from 8 to $3.5 \mathrm{GeV}$ and the beam current is increased from 1.1 to $9.4 \mathrm{~A}$. The vacuum pressure in beam chambers will be increased from 1 to $5 \mathrm{nTorr}$ due to the large stored current. All factors contribute to an enhancement of the ion effects. The ion instability for high charge mode is about 19 times stronger than present KEKB. It will be 24 times stronger in the low charge mode. Low charge mode is chosen in the current design. The ion effects can be strong enough to degrade the luminosity.

A systematic scan of the beam filling pattern has been done in order to optimize the filling pattern. Figure 16 shows the growth rate for various beam filling patterns with a partial pressure of $\mathrm{CO}^{+} 1 \mathrm{nTorr}$. It clearly shows the mitigation of instability with a longer train gap and shorter train. The simulated growth rate for a fixed bunch-train gap is proportional to the number of bunches per train. There is a similar total bunch number $n_{b}$ for these filling patterns. As a result, the ion density near the beam, and therefore the instability growth rate, is approximately proportional to the number of bunches per bunch train $M$ :

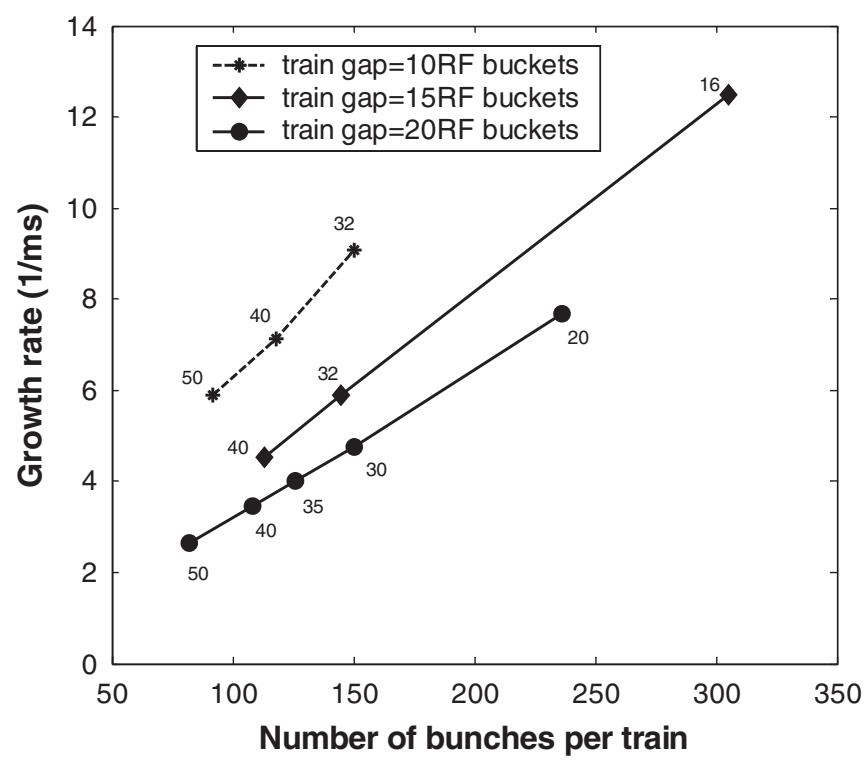

FIG. 16. Instability growth rate in SuperKEKB (high charge option) as a function of the number of bunches per bunch train for different train gaps of 10, 15, and 20 buckets. The pressure is $1 \mathrm{nTorr}$ of $\mathrm{CO}^{+}$. The number of bunch trains is also shown in the plot.

$$
f_{\text {gap }}=\frac{1}{n_{b}} \frac{M}{1-\exp \left(-\tau_{\text {gap }} / \tau_{\text {diffusion }}\right)} \propto M .
$$

The simulation agrees well with the above relation.

It is more interesting that the simulated growth rate for different beam filling patterns in SuperKEKB follows the scaling law,

$$
\frac{1}{\tau_{\text {Super }_{\text {КЕKB }}}}=0.59 \frac{r_{e} c \bar{\beta}_{y} \rho_{i c}}{\gamma} .
$$

Here $\rho_{i c}$ is the maximum ion density near the beam $\rho_{i c} \approx k_{y} \rho_{\infty}$. An average betatron function is used. The factor $Q$, which includes both the nonlinear force and optics effects, is included in the coefficient 0.59 . Comparing this scaling law with Eq. (34), it can be concluded that the factor $Q$ in SuperKEKB is smaller than 2, which indicates that the frequency spread due to variation of the beam size along the ring provides an effective damping of the instability.

\section{Observation of beam-ion instability in SPEAR3 with multibunch train beam filling pattern}

SPEAR3 has a circumference of $234 \mathrm{~m}$ with a harmonic number of 372 . The normal beam filling pattern is a single bunch train with 280 bunches followed by a gap of $92 \mathrm{rf}$ buckets spacing. The natural emittance is $10 \mathrm{~nm}$ and there is a low vertical emittance of $14 \mathrm{pm}$. Vertical coupled bunch instability has been observed at nominal vacuum pressure with a single bunch-train filling pattern when the beam current is larger than $200 \mathrm{~mA}$. The vertical instability becomes stronger when there is a vacuum pressure rise by partially turning off the ion pumps. The instability also varies with vertical emittance. The couple bunch instability driven by impedance, for instance, the resistive wall impedance, does not change with vacuum pressure and beam size. All these observations directly confirm that the observed instability is caused by the ions in the vacuum. Moreover, the observed frequency of the sideband agrees with the analysis and it increases with beam current as expected. The observed amplitude of vertical oscillation of the electron beam saturates at a few rms beam size as shown later, which is also one important character of the beam-ion instability.

The beam-ion instability strongly depends on both total vacuum pressure and vacuum component. Different ion species have different ionization cross section and have different dynamics, for instance, the trapping condition and oscillation frequency. The dominant gas species in SPEAR3 are hydrogen, methane, water, carbon monoxide, and carbon dioxide. Among them, the hydrogen ion has the largest frequency about $70 \mathrm{MHz}$ due to its small mass number and carbon dioxide has low frequency order of $10 \mathrm{MHz}$. Figure 17 shows calculated oscillation frequency of $\mathrm{CO}^{+}$along the SPEAR3 ring. A constant emittance is used in the calculation. The vertical emittance in the real 


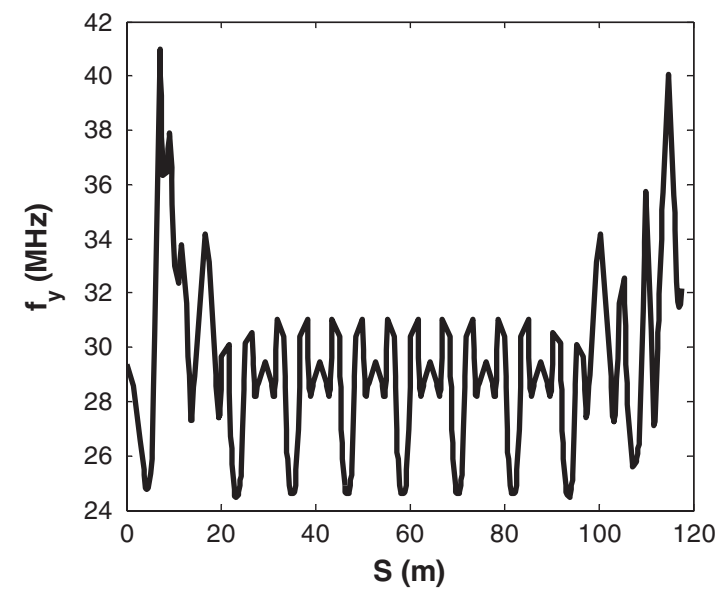

FIG. 17. Calculated oscillation frequency of $\mathrm{CO}^{+}$along the SPEAR3 ring. The beam current is $500 \mathrm{~mA}$ and bunch number is 280.

machine varies along the ring. Table II shows the ions species in the SPEAR3 vacuum chamber from two vacuum gauges. The total pressure ranges from 0.1 to 0.5 nTorr and varies in different regions along the ring.

Figure 18 shows the measured beam's vertical oscillation amplitude with a single bunch-train filling pattern at $200 \mathrm{~mA}$ beam current. The number of bunches is 280 . The oscillation amplitude increases along the bunch train. Bunches in the tail of the bunch train have larger amplitude. The amplitude saturates at $14 \mu \mathrm{m}$, which is the order of beam size. All these are characters of beam-ion instability. Figure 19 shows the observed vertical lower sideband from the beam spectrometer for a different length of bunch-train gap at $200 \mathrm{~mA}$ beam current with a single bunch-train filling pattern. The instability becomes stronger when the train gap is reduced. The frequency of unstable modes also becomes broader.

We have observed the vertical lower sideband for different beam filling patterns with a total beam current of $500 \mathrm{~mA}$ as shown in Fig. 20. It clearly shows that the multibunch train filling pattern can significantly mitigate the instability. The instability becomes weak with six bunch-trains filling pattern and it is slightly better than the four bunch-train case. One can compare the calculated frequency of $\mathrm{CO}^{+}$shown in Fig. 17 with the frequency of unstable modes. The observed frequency of unstable modes agrees with the analysis overall, although it is

TABLE II. Ion species in SPEAR3 vacuum chamber.

\begin{tabular}{lcc}
\hline \hline Ion species & Mass number & Percentage in vacuum \\
\hline $\mathrm{H}_{2}$ & 2 & $48 \%$ \\
$\mathrm{CH}_{4}$ & 16 & $5 \%$ \\
$\mathrm{H}_{2} \mathrm{O}$ & 18 & $16 \%$ \\
$\mathrm{CO}$ & 28 & $14 \%$ \\
$\mathrm{CO}_{2}$ & 44 & $17 \%$ \\
\hline \hline
\end{tabular}

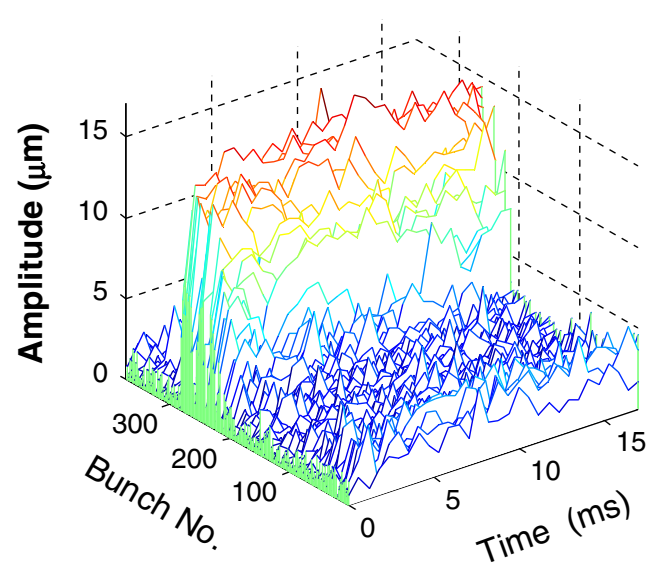

FIG. 18. Measured beam's vertical oscillation amplitude in SPEAR3 with single bunch-train filling pattern. The number of bunches is 280 and the total beam current is $200 \mathrm{~mA}$.

difficult to tell what types of ions drive each unstable mode because the frequencies of different ions overlap each other due to the variation of beam size along the ring. Figure 21 shows the simulated ion density for a different beam filling pattern with a total vacuum pressure of $0.5 \mathrm{nTorr}$. It clearly shows the reduction of the density of trapped ions by the multibunch train filling pattern. The mitigation is very effective with up to four bunch trains. It does not improve much with six bunch trains, which agrees with the observation shown in Fig. 20. Figure 22 shows the simulated vertical oscillation amplitude for the six bunchtrain beam filling pattern with a total beam current of $500 \mathrm{~mA}$. The total vacuum pressure is $0.5 \mathrm{nTorr}$ with ion species shown in Table II. The amplitude saturates at a few of rms beam size (the vertical beam size is $13 \mu \mathrm{m}$ here.) The exponential growth time is $1.62 \mathrm{~ms}$, which is shorter than the radiation damping time of $5.3 \mathrm{~ms}$. SPEAR3 runs with a six bunch-train filling pattern after this study and the beam-ion instability is totally suppressed by a six bunchtrain filling pattern at present beam current $300 \mathrm{~mA}$. In $200 \mathrm{~mA}$ operation mode, the sideband completely disappears with a two bunch-train filling pattern. The simulated growth rate with the assumed vacuum pressure is slightly larger (less than factor 2) than the observation, which indicates the average vacuum pressure in SPEAR3 is lower than 0.5 nTorr.

Figure 23 compares the observed vertical oscillation amplitude at each sideband estimated from the low sideband for different vertical emittance with a six bunch-train beam filling pattern. The vertical emittance is increased by a factor of 10 when all skew quadrupole magnets are off. It is clearly shown that the observed frequency of the sidebands becomes lower and the peak amplitude of the sidebands also decreases when the vertical emittance increases. These qualitatively agree with the theory of beam-ion instability and the instability driven by traditional impedance does not change with beam emittance. 

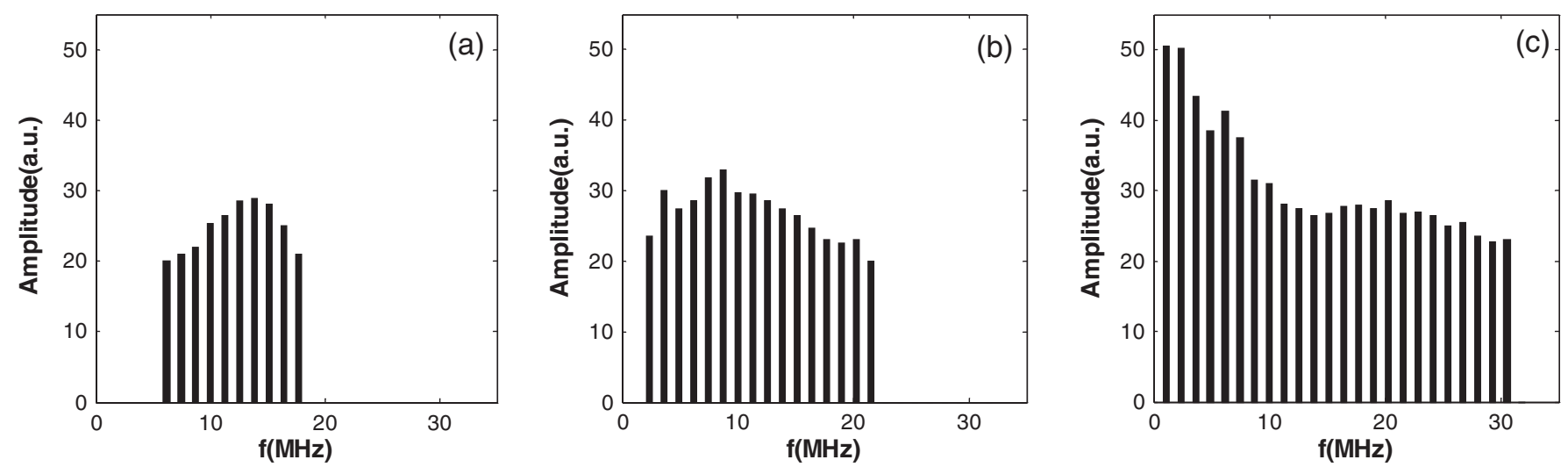

FIG. 19. Measured vertical lower sideband for different train gap length (a) $189 \mathrm{~ns}$, (b) $38 \mathrm{~ns}$, and (c) 17 ns. The beam filling pattern is a single bunch train with total beam current of $200 \mathrm{~mA}$.
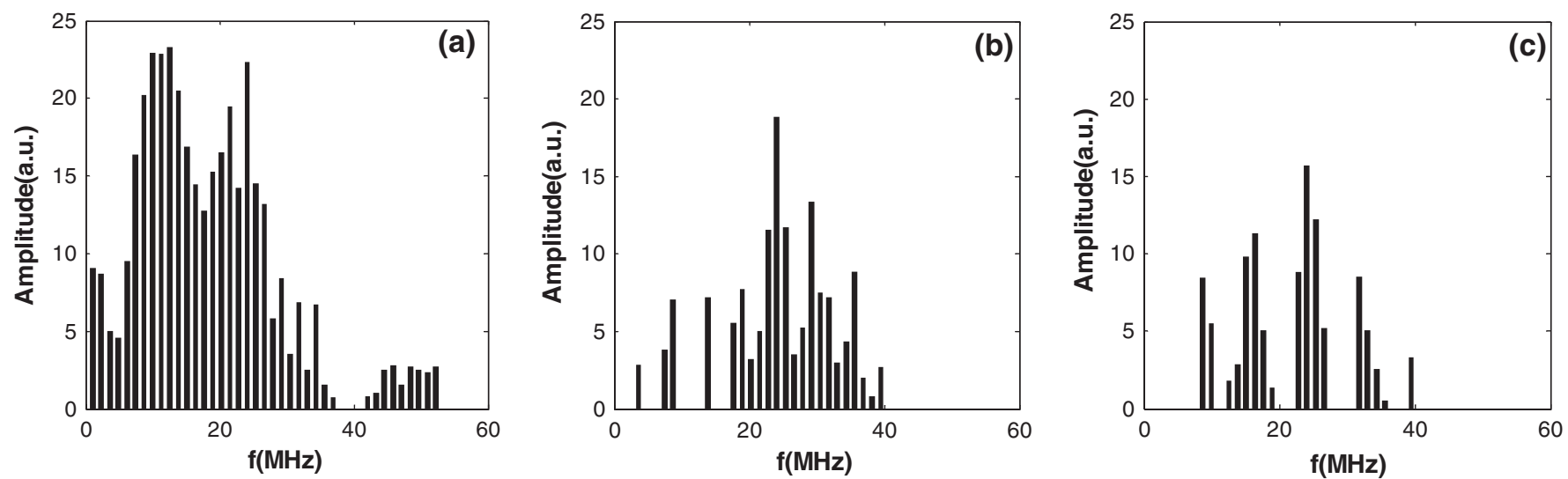

FIG. 20. Measured beam's vertical lower sideband for different beam filling patterns: (a) one bunch train; (b) four bunch train; (c) six bunch train. The total beam current is $500 \mathrm{~mA}$ with total bunch number of 280 in all cases.

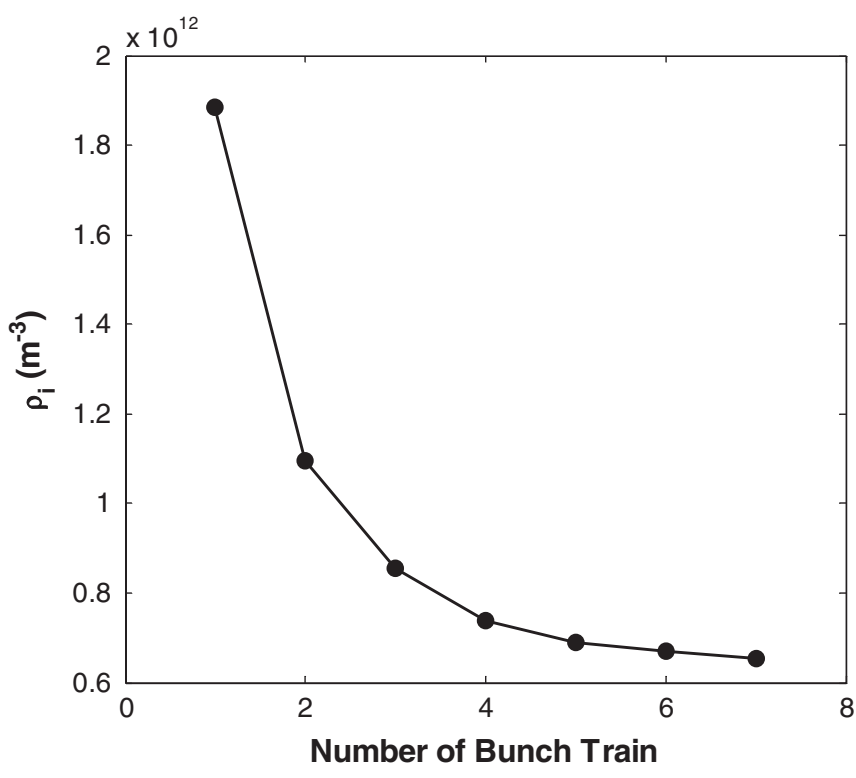

FIG. 21. Dependence of density of trapped ions on the beam filling pattern by simulation. The total beam current is $500 \mathrm{~mA}$ with total bunch number of 280 in all cases.

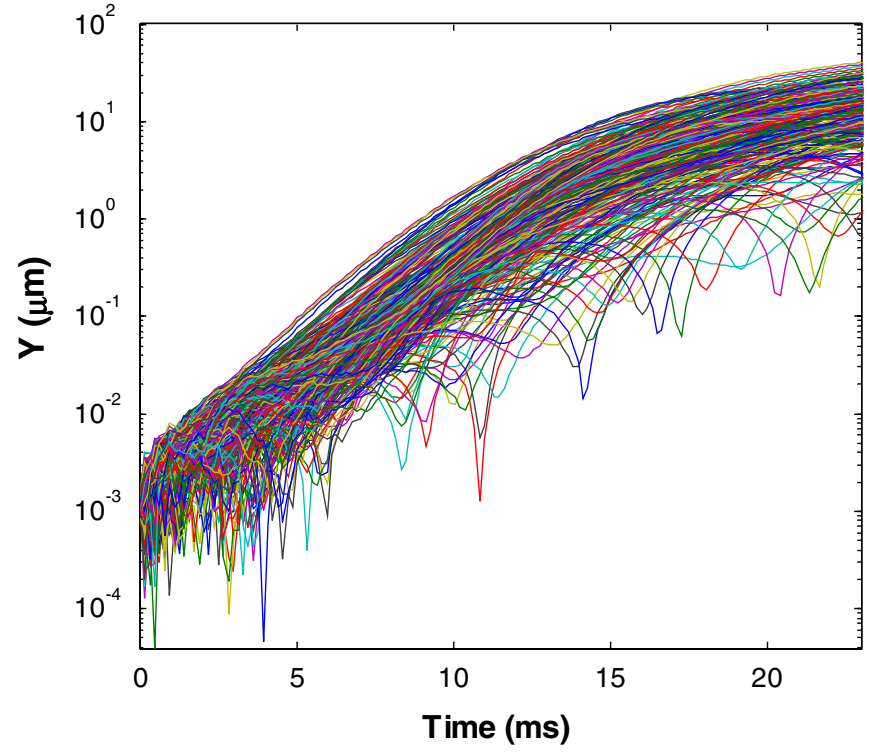

FIG. 22. Simulated vertical beam instability in SPEAR3. The beam consists of a six bunch train with a total beam current of $500 \mathrm{~mA}$. The total vacuum pressure is $0.5 \mathrm{nTorr}$ with ion species shown in Table II. 


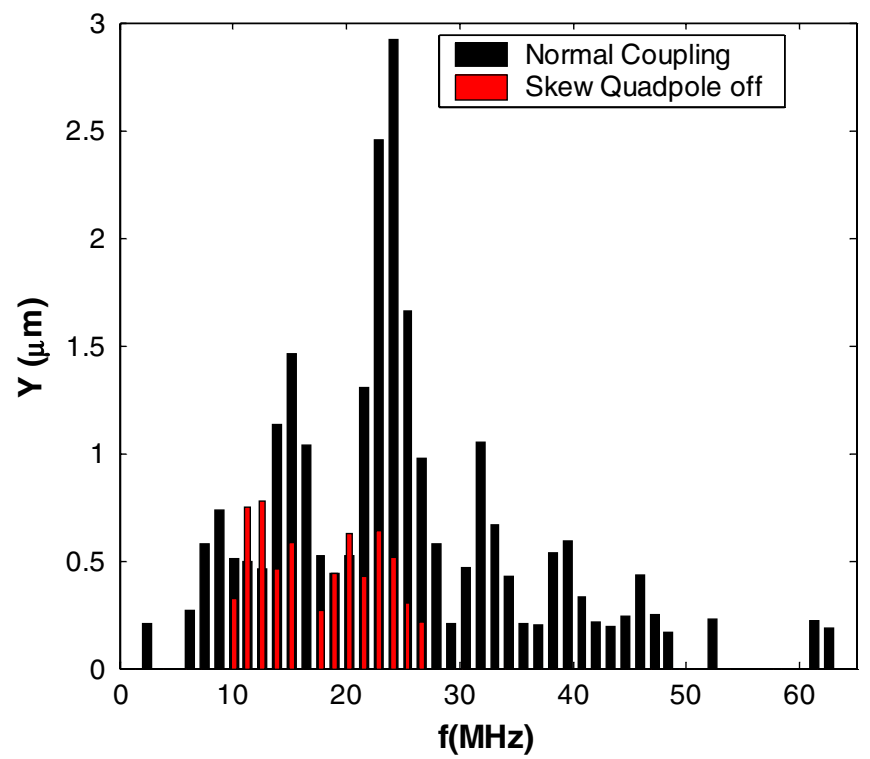

FIG. 23. Measured beam's vertical lower sideband for different vertical emittance with a six bunch-train beam filling pattern. The total beam current is $500 \mathrm{~mA}$ and total bunch number is 280 . The vertical emittance is increased by a factor of 10 when all skew quadrupole magnets are off.

A better vacuum can directly reduce the growth rate of the instability; however, the improvement is limited and the cost can be prohibitive. A bunch-by-bunch feedback can be used to completely suppress the instability if a multibunch train is not effective enough. There is no bunch-by-bunch feedback system in SPEAR3. Fortunately, we could have one chance to test one feedback system from Dimtel Inc. [32]. Figure 24 shows the observed damping of the vertical instability by the feedback system. The beam consists of a six bunch train with total beam current $448 \mathrm{~mA}$. The feedback is initially turned off and the beam has maximum amplitude of $35 \mu \mathrm{m}$. The amplitude is damped to the $\mu \mathrm{m}$ level when the feedback is turned on. Since we only have one chance to study the feedback system, the effects of the feedback system, such as noise and the required voltage, have not been studied in detail.

Simulation shows that a feedback with a damping time shorter than the exponential growth time is required. A semianalytical study also confirms that a feedback with a damping time of the same level as exponential growth time seems enough to damp the instability to the noise level [33]. When a feedback system with a damping time of a few tens of turns is required, there may be issues associated with noise within the feedback system itself. Specifically, noise in the pickup or amplifier may be transferred to the kicker, which then induces some jitter on the beam. The net result of the feedback is that the beam will reach certain rms oscillation amplitude which is determined by the feedback damping and noise [34].

The growth rate of single bunch-train instability increases along the bunch train. Therefore it is expected that a slow feedback can damp bunches in the head of the bunch train first and then gradually stabilizes the whole bunch train. This is only true for the ideal case without any jitter and errors. In reality, a slow feedback with a damping time longer than the exponential growth time can only damp the nonlinear instability and reduces the beam's oscillation amplitude to the order of beam size. Therefore, the beam seems stable even with a slow feedback. However, the remaining oscillation can still quietly degrade the beam quality.
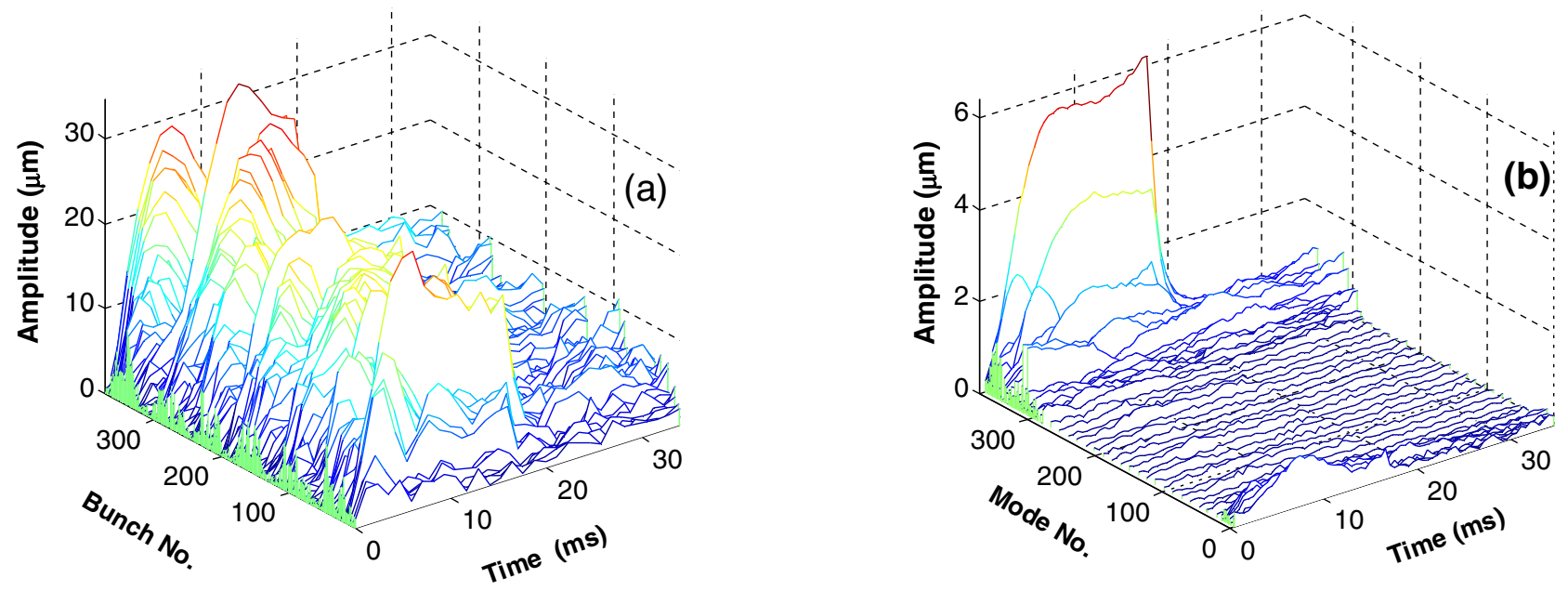

FIG. 24. Measured beam's vertical oscillation amplitude (a) and unstable mode (b) when the bunch-by-bunch feedback is on and off. The feedback is initially turned off and turned on around $20 \mathrm{~ms}$. The beam consists of a six bunch train with total beam current of $448 \mathrm{~mA}$. 


\section{COHERENT TUNE SHIFT}

In this section, we briefly discuss another important aspect: coherent tune shift due to ions. The focusing force of trapped ions causes a positive tune shift to the electron bunches:

$$
\Delta \nu_{y}=\frac{\beta_{y}}{4 \pi} \frac{\partial \Delta y_{e}^{\prime}}{\partial y_{e}}=\frac{r_{e}}{3 \pi \gamma} \int_{\text {ion trapped region }} \frac{\beta_{y} \lambda_{\text {ion }}}{\sigma_{y}\left(\sigma_{x}+\sigma_{y}\right)} d s .
$$

If the tune shift is too large, it may degrade the performance of the accelerator [33]. Similar to the instability growth rate, the tune shift is also proportional to the ion density. Therefore, the tune shift of electron bunches for a single bunch-train filling pattern linearly increases along the bunch train. The ion cloud is assumed to be stable in the derivation of the above equation. The simulated tune shift is a perfect linear function of bunch number when the beam is stable and agrees well with analysis as shown in Fig. 25. Each time only one test electron bunch oscillates under the ion cloud force. Other bunches are stabilized by a bunchby-bunch feedback system. In this way we can get the tune of the test bunch in each simulation.

When the beam is unstable, the tune shift depends on the coupled motion between the electron beam and ion cloud. Figure 26 shows the dependence of the tune shift on the coupled motion in the linear regime where the amplitude of beam oscillation is smaller than the beam size. For example, when all electron bunches have the same phase ( 0 mode), the ion cloud does not oscillate coherently and causes the smallest tune shift. On the other hand, when the

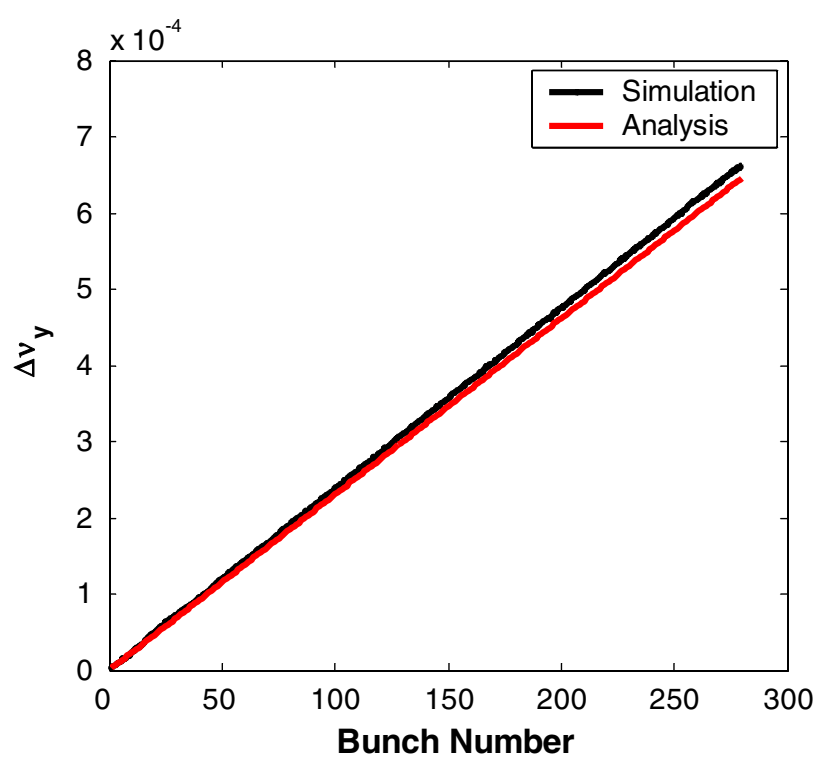

FIG. 25. Simulated vertical tune shift along the bunch train for SPEAR3 beam with a partial pressure of $1 \mathrm{nTorr} \mathrm{CO}^{+}$. The beam has a single bunch train with total bunch number of 280. A fast bunch-by-bunch feedback is applied to stabilize the beam. When feedback is turned off, the tune shift varies with time.

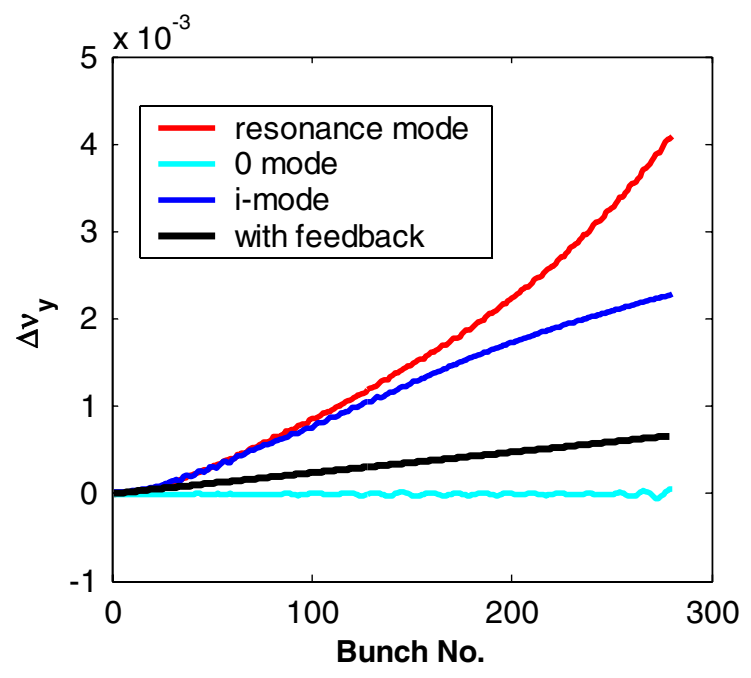

FIG. 26. Dependence of vertical tune shift along the bunch train on the coupled motion in the linear regime. Here $i$ mode is one of the modes other than 0 and resonance modes.

$k_{\mathrm{th}}$ electron bunches has initial phase $\phi_{k}(t=0)=\phi_{0}+$ $\omega_{i} k S_{b} / c$ (resonance mode), the ion cloud oscillates resonantly with the electron beam and causes the largest tune shift to the electron beam. It can be a few times larger than that given by Eq. (44).

However, the tune shift is quite different in the nonlinear regime when the amplitude of electron beam is larger than its transverse beam size where there is a strong nonlinear force. Meanwhile, the instability significantly changes both the distribution and density of the ion cloud. Simulation shows that the tune shift of the resonance mode becomes comparable with Eq. (44) in the nonlinear region. Note that the observed tune shift without feedback is in the nonlinear regime, which should not be compared with Eq. (44). The tune shift with an unstable beam is complicated and will be addressed separately elsewhere.

\section{CONCLUSIONS}

The suppression of beam-ion instability using a multibunch train filling pattern is systematically studied. It is an effective mitigation for high intensity beams with a low emittance. Simple formulas are derived for the buildup of ion cloud [Eq. (31)] and growth rate of the instabilities with a general beam filling pattern [Eq. (34)]. Our analysis agrees well with simulation.

Both the distribution and electric field of trapped ions are benchmarked with a numerical approach. The distribution of ion cloud has a sharp peak near the center and its dimension is smaller than the electron bunch from which the ions are born. However, the electric field of the ion cloud closely approximates that of a Gaussian distribution with $\sigma_{i}^{2} \approx \sigma_{e}^{2} / 2$.

A wakefield model is introduced to study the beam-ion instability in the exponential growth regime. The simulated 
wake agrees reasonably with the analysis model. The total wakefield of an ion cloud along the whole ring is proportional to the ion density and the circumference of the ring. The wakefield of ion cloud with a flat beam is inversely proportional to the 1.5 power of the product of horizontal and vertical beam size. Therefore, the beam-ion instability can be very strong for future ultrasmall emittance rings.

We briefly discuss the beam-ion instability growth rate with different beam filling patterns: uniform beam filling, single bunch-train filling pattern, and multibunch train filling pattern. It is interesting that the maximum exponential growth rate in all cases has the same form and is proportional to the average ion density near the beam seen by the electron bunches.

Both simulation and experiment found that the ion cloud exhibits exponential decay during the bunch-train gap with an exponential time constant of the order of the ion's oscillation period. This phenomenon can be explained by the characteristics of the motion of the ion particle. This feature makes the multibunch train filling pattern a very effective way to reduce the ion density for an electron ring with high beam current and low emittance. The reduction of the ion density can be up to 2 orders of magnitude. Several examples are given and there is good agreement among analysis, simulation, and experiment. The observation from SPEAR3 also directly confirms the effectiveness of the multibunch train filling pattern on suppression of the beam instability. The optimized beam filling pattern varies with the beam, for instance, 183 bunch trains for PEPX, 20 bunch trains for NSLSII, and six bunch trains for SPEAR3. After the experiment, SPEAR3 runs with a six bunch-train filling pattern.

A bunch-by-bunch feedback with a damping time shorter than the exponential growth time is required to completely damp the instability. A slow feedback can only damp the nonlinear instability and reduce the beam oscillation amplitude to the order of the beam size. The experiment in SPEAR3 shows that the feedback reduces the beam oscillation amplitude below one-tenth of the beam size.

The trapped ions induce a positive coherent tune shift. With an unstable beam, the tune shift is sensitive to the coupled mode of electron beam and it dynamically changes with time. When the beam is stabilized by a feedback system, the ion-induced tune shift agrees well with the analysis.

\section{ACKNOWLEDGMENTS}

We would like to acknowledge the SPEAR3 team for its help on the SPEAR3 observations, with special thanks to J. Safranek for his support and contributions. We also thank D. Teytelman for providing the results of feedback system. L. Wang would like to thank A. Chao, G. Stupakov, K. Ohmi, and M. Takao for fruitful discussions, M. Boland at AS, and A. Streun at SLS for providing the parameters of the rings.

\section{APPENDIX: TRANSVERSE DISTRIBUTION OF IONS}

The ion's distribution without beam instability is discussed here. The ions are born from the electron bunches in our case. Therefore they have the same distribution as the electron bunch when they are born. Then the ions move under the potential of electron bunches. The self-space charge of ions is neglected here. The electric field of a bi-Gaussian beam is

$$
\begin{aligned}
& E_{x}=\frac{e N_{e}}{2 \pi \varepsilon_{0}} x \int_{0}^{\infty} \frac{\exp \left[-\frac{x^{2}}{2 \sigma_{x}^{2}+t}-\frac{y^{2}}{2 \sigma_{y}^{2}+t}\right]}{\left(2 \sigma_{x}^{2}+t\right)^{3 / 2}\left(2 \sigma_{y}^{2}+t\right)^{1 / 2}} d t \\
& =\frac{e N_{e}}{4 \pi \varepsilon_{0}} \sqrt{\frac{2 \pi}{\sigma_{e, x}^{2}-\sigma_{e, y}^{2}}} \operatorname{Re}[f(x, y)] \text {, } \\
& E_{y}=\frac{e N_{e}}{2 \pi \varepsilon_{0}} y \int_{0}^{\infty} \frac{\exp \left[-\frac{x^{2}}{2 \sigma_{x}^{2}+t}-\frac{y^{2}}{2 \sigma_{y}^{2}+t}\right]}{\left(2 \sigma_{x}^{2}+t\right)^{1 / 2}\left(2 \sigma_{y}^{2}+t\right)^{3 / 2}} d t \\
& =\frac{e N_{e}}{4 \pi \varepsilon_{0}} \sqrt{\frac{2 \pi}{\sigma_{e, x}^{2}-\sigma_{e, y}^{2}}} \operatorname{Im}[f(x, y)], \\
& f(x, y)=w\left(\frac{x+i y}{\sqrt{2\left(\sigma_{e, x}^{2}-\sigma_{e, y}^{2}\right)}}\right) \\
& -\exp \left(-\frac{x^{2}}{2 \sigma_{e, x}^{2}}-\frac{y^{2}}{2 \sigma_{e, y}^{2}}\right) w\left(\frac{x \frac{\sigma_{e, y}}{\sigma_{e, x}}+i y \frac{\sigma_{e, x}}{\sigma_{e, y}}}{\sqrt{2\left(\sigma_{e, x}^{2}-\sigma_{e, y}^{2}\right)}}\right) \text {, } \\
& w(z)=e^{-z^{2}}\left[1+\frac{2 i}{\sqrt{\pi}} \int_{0}^{z} e^{t^{2}} d t\right], \quad z=x+i y,
\end{aligned}
$$

where $\sigma_{e, x, y}$ is the transverse root mean square (rms) beam size. When $x<\sigma_{e, x}$ and $y<\sigma_{e, y}$, there is a linear field

$$
\left[\begin{array}{l}
E_{x} \\
E_{y}
\end{array}\right]=\frac{e N_{e}}{2 \pi \varepsilon_{0} \sigma_{e, x, y}\left(\sigma_{e, x}+\sigma_{e, y}\right)}\left[\begin{array}{l}
x \\
y
\end{array}\right] .
$$

The motion of ions in horizontal and vertical directions is decoupled in the linear regime. Here we consider the horizontal motion only. With the field given by Eq. (A5), the motion equation of an ion can be written in the form

$$
\ddot{x}+\omega_{x 0}^{2} x=0 .
$$

A good approximation is obtained by assuming that the ions are born at rest since the beam's potential (up to $\mathrm{keV}$ level) is much larger than the ions' thermal energy (less than $1 \mathrm{eV}$ ). Therefore the solution of horizontal motion is

$$
x(t) \approx x_{0} \sin \left(\omega_{x 0} t+\phi_{0}\right),
$$

where $x_{0}$ is the ion's oscillation amplitude, which is approximately the position at its birth. $\varphi_{0}$ is the ion's initial 


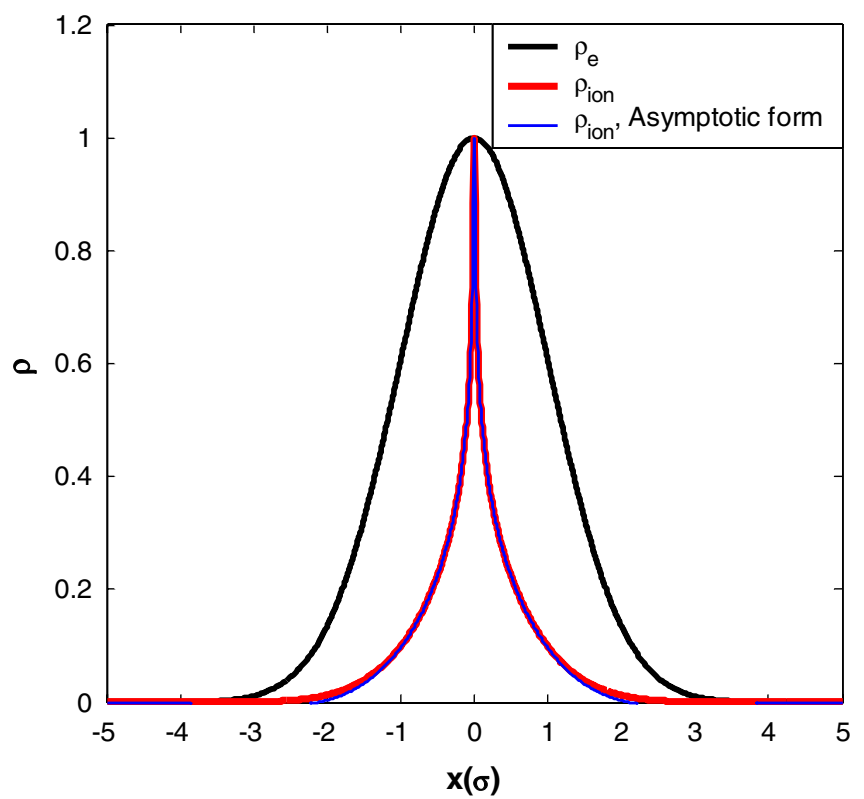

FIG. 27. One-dimensional distribution of an ion cloud and the electron bunch. The ion distribution with asymptotic form also is shown. The horizontal axis is in the unit of the rms beam size.

phase when the ions are born, which has a uniform distribution due to the random generation of ions (time). Therefore, the ions born at $x_{0}$ have the following distribution in $x$ :

$$
f\left(x, x_{0}\right)=\frac{2}{\pi \sqrt{x_{0}^{2}-x^{2}}}, \quad\left(x<x_{0}\right) .
$$

The initial position of ions $x_{0}$ has a Gaussian distribution with the same dimension as the electron bunch,

$$
\rho_{0}\left(x_{0}\right)=\frac{1}{\sqrt{2 \pi} \sigma_{e, x}} \exp \left(-\frac{x_{0}^{2}}{2 \sigma_{e, x}^{2}}\right) .
$$

Therefore the distribution of ions at the equilibrium becomes

$$
\begin{aligned}
\rho(x) & =\int_{x}^{\infty} f\left(x, x_{0}\right) \rho_{0}\left(x_{0}\right) d x_{0} \\
& =\frac{1}{\pi \sqrt{2 \pi} \sigma_{e, x}} e^{-\left(x^{2} / 4 \sigma_{e, x}^{2}\right)} K_{0}\left(\frac{x^{2}}{4 \sigma_{e, x}^{2}}\right),
\end{aligned}
$$

where $K_{0}$ is the modified Bessel function of the second kind and its asymptotic form is

$$
K_{0}(x) \approx \begin{cases}-\ln \frac{x}{2}-\gamma_{c} & x \rightarrow 0 \\ \sqrt{\frac{\pi}{2 x}} e^{-x} & x \rightarrow \infty,\end{cases}
$$

where the constant $\gamma_{c}=-\int_{0}^{\infty} e^{-x} \ln x d x=0.5772156649$. With the asymptotic form, the ion distribution near the beam center is

$$
\rho(x) \approx-\frac{1}{\pi \sqrt{2 \pi} \sigma_{e, x}} e^{-\left(x^{2} / 4 \sigma_{e, x}^{2}\right)}\left[\ln \left(\frac{x^{2}}{8 \sigma_{e, x}^{2}}\right)+\gamma_{c}\right] .
$$

Equations (A10) and (A12) gives the one-dimensional ion distribution at the equilibrium. The same result of Eq. (A10) was first given by Tavares [15]. But the asymptotic form in [15] is incorrect.

Figure 27 shows the horizontal distributions of an electron bunch and the ions born from the electron bunch. The ion distribution has a sharp peak near the center and its dimension is smaller than the electron bunch. Surprisingly, the asymptotic form agrees very well within two rms beam size, where most of the ions are trapped. Therefore, the asymptotic form is good enough to express the ion distribution.

[1] J. Byrd et al., Phys. Rev. Lett. 79, 79 (1997).

[2] J. Y. Huang et al., Phys. Rev. Lett. 81, 4388 (1998).

[3] M. Kwon et al., Phys. Rev. E 57, 6016 (1998).

[4] K. Y. Ng and M. Popovic, Report No. FERMILAB-FN0712, 2001.

[5] S. J. Werkema, K. D. Fullett, and P. Zhou, in Proceedings of the Particle Accelerator Conference, Washington, DC, 1993 (IEEE, New York, 1993), p 3309.

[6] T. O. Raubenheimer and F. Zimmermann, Phys. Rev. E 52, 5487 (1995).

[7] G. V. Stupakov, T. O. Raubenheimer, and F. Zimmermann, Phys. Rev. E 52, 5499 (1995).

[8] G. V. Stupakov, KEK Proceedings 96-6, 1996, 243.

[9] K. Ohmi, Phys. Rev. E 55, 7550 (1997).

[10] E. Bozoki and D. Sagan, Nucl. Instrum. Methods Phys. Res., Sect. A 340, 259 (1994).

[11] M. Zobov, JINST 2, P08002 (2007).

[12] L. Wang, T. O. Raubenheimer, and A. Wolski, in Proceedings of the 10th European Particle Accelerator Conference, Edinburgh, Scotland, 2006 (EPS-AG, Edinburgh, Scotland, 2006), p. 2155.

[13] G. Xia and E. Elsen, Nucl. Instrum. Methods Phys. Res., Sect. A 593, 183 (2008).

[14] E.S. Kim and K. Ohmi, Jpn. J. Appl. Phys. 48, 086501 (2009).

[15] P. F. Tavares, Report No. CERN PS/92-55 (LP) 1992.

[16] K. Ohmi, F. Zimmermann, and E. Perevedentsev, Phys. Rev. E 65, 016502 (2001).

[17] K. Ohmi and F. Zimmermann, Phys. Rev. Lett. 85, 3821 (2000).

[18] Alexander W. Chao, Physics of Collective Beam Instabilities in High Energy Accelerators (Wiley, New York, 1993).

[19] L. Wang, Y. Cai, and T. O. Raubenheimer, in Proceedings of the 22nd Particle Accelerator Conference (PAC'07), Albuquerque, USA, 2007, p. 4240

[20] R. Hettel et al., in Proceedings of the 23rd Particle Accelerator Conference, Vancouver, Canada, 2009 (IEEE, Piscataway, NJ, 2009), p. 2297.

[21] H.S. Kang et al., in Proceedings of the 10th European Particle Accelerator Conference, Edinburgh, Scotland, 2006 (Ref. [12]), p. 2771.

[22] R. Nagaoka et al., in Proceedings of the 16th IEEE International Pulsed Power Conference, Albuquerque, NM (Ref. [19]), p. 2019. 
[23] B. Jiang et al., Nucl. Instrum. Methods Phys. Res., Sect. A 614, 331 (2010).

[24] D. J. Peake et al., in Proceedings of the 23rd Particle Accelerator Conference, Vancouver, Canada, 2009 (Ref. [20]), p. 4105.

[25] R. A. Bosch, Nucl. Instrum. Methods Phys. Res., Sect. A 450, 223 (2000).

[26] S. Heifets et al., Report No. SLAC-PUB-12959, 2007.

[27] M. Takao et al., in Proceedings of the 8th European Particle Accelerator Conference, Paris, 2002 (EPS-IGA and CERN, Geneva, 2002), p. 1562.

[28] D. Sagan and A. Temnykh, Nucl. Instrum. Methods Phys. Res., Sect. A 344, 459 (1994).
[29] H. Grote and F. Schmidt, in Proceedings of the 20th Particle Accelerator Conference, Portland, OR, 2003 (IEEE, New York, 2003), p. 3497.

[30] Y. Cai, A. W. Chao, and S. I. Tzenov, Phys. Rev. ST Accel. Beams 4, 011001 (2001).

[31] M. Bassetti and G. A. Erskine, Report No. CERN-ISR$\mathrm{TH} / 80-06$.

[32] http://www.dimtel.com/.

[33] H. Fukuma and L. Wang, in Proceedings of ECLOUD'07, Daegu, Korea, 2007 (Report No. SLAC-PUB-12757, 2007).

[34] A. W. Chao and G. V. Stupakov, KEK Proceedings No. 9717, 1997, p. 110. 